\title{
Cytotoxic Compounds Derived from Marine Algicolous and Spongicolous Endophytic Fungi: A Review
}

\author{
Nadia Tasnim Ahmed ${ }^{1}$, Sadia Noor ${ }^{1}$, Md Mustafizur Rahman ${ }^{2}$ and \\ Md. Abdul Mazid ${ }^{1}$ \\ ${ }^{1}$ Department of Pharmaceutical Chemistry, University of Dhaka, Dhaka-1000, Bangladesh \\ ${ }^{2}$ Pharmacy Discipline, Khulna University, Khulna-9208, Bangladesh
}

(Received: August 26, 2021; Accepted: December 20, 2021; Published (web): December 26, 2021)

\begin{abstract}
Endophytes have gained particular interest in the search of potential pharmaceutical candidates for a long time due to their diversity, species richness and bioprospecting nature. They generally produce the essential metabolites for their expansion inside the plant which is involved in various biotransformation processes of utilizing host nutrients and cell components to continue microbial growth, sustenance, and reproduction. In above processes, they produce a huge amount of both structurally and functionally diverse secondary metabolites for maintaining an effective symbiosis with hosts. These compounds are proven to have significant bioactive properties like antibacterial, antifungal, antiviral, anti-inflammatory, antioxidants, antitumor activities. Despite the proven significance, a little is exploited so far about endophytes. Particularly marine fungal endophytes which are the centre of attention in this review have gained much less importance. Due to unique environmental feature, fungal endophytes derived from marine environment offer vast diversity in different bioactive secondary metabolites. This review has focused on algicolous endophytes and bioactive secondary metabolites discovered during the last two decades. Particular importance has been given to cytotoxic and antimicrobial metabolites. Due to intensive studies during last several years, an extensive number of publications are now available on cytotoxic compounds derived from endophytic fungi of marine algicolous and spongicolous origin that have been summarized in this review.
\end{abstract}

Key words: Endophytic fungi, Cytotoxic compounds, Bioactive secondary metabolites, Marine algicolous and spongicolous endophytes

\section{Endophytes and Endophytism}

Endophytes are either bacterial (including actinomycetes) or fungal microorganism. These endophytes spend the whole or part of their life cycle living inter- and/or intra-cellularly inside the healthy tissues of the host plant. ${ }^{1}$ They are non-organ specific, can invade any plant organs and reside beneath the epidermal cells of plants. ${ }^{2-4}$ There is no apparent evidence of their existence in plants because they neither cause any noticeable tissue damage nor produce any symptoms. ${ }^{5}$ They may be present ubiquitously in each and every healthy plant usually more than one in each flora through a symbiotic association with the host. ${ }^{6,10}$ In general, bacteria, archaebacteria, fungi, algae, mycoplasma all can act

Correspondence to: Md. Abdul Mazid

Email: ma.mazid@du.ac.bd

Dhaka Univ. J. Pharm. Sci. 20(2): 247-265, 2021 (December) DOI: https://doi.org/10.3329/dujps.v20i2.57175 as endophytes. They are prolific sources of bioactive metabolites which they produce via direct association with their hosts. ${ }^{10}$ Our main focus for this review is fungi or mycoendophytes derived from or associated with marine sources i.e., macroalgae, and bioactive secondary metabolites obtained from them.

\section{Algicolous Fungi \\ Marine algae usually contribute one-third of well known higher fungal endophytes. For more than 2000 years, macroalgae have been widely exploited traditionally in China and ancient Egypt as folk medicine. Most algicolous marine fungi are Ascomycetes. For adaption with the constant environmental stress of marine atmosphere such as frequent change in moisture and salt concentration, sunlight exposure for prolonged time, tide alteration, abundant microorganisms and herbivore insects, etc.,}


it apparently looks like that both marine algae and their endophytic symbionts could be a significant source of secondary metabolites having biological activities. $^{7}$

\section{Isolation and cultivation of fungi from marine organisms}

As a general approach, surface sterilizaiton techniques are employed to isolate endophytes from marine algae. Initially, epiphytes inhabited in the outer surface of algae are removed by this sterilization process. The surface sterilization are done in different ways depending on microbial species and/or host tissue types. Several mechanical processes such as ultrasonic bath sonication, ultrasonic probe sonication, use of beads and vortexing; using enzymes such as proteinase $\mathrm{K}$, lysozyme; and chemicals such as bleaching agent, ethanol, alkaline lysis buffer, UNSET buffer, CTAB buffer, bactericidal cleanser, etc. have been used by scientists to eliminate epiphytes completely from the sample surface as per suitability. ${ }^{8}$ Researcher usually choose healthy and disease free fresh algae for isolation purpose. This is important to avoid isolation of localized pathogens and saprophytic microbes. ${ }^{9,10}$ Moreover, to eleminate contamination by air microspores, samples after collection are needed to be processed immediately or stored at $4^{\circ} \mathrm{C}$ in case of delayed processing. ${ }^{11,12}$ The most commonly used approaches followed reserachers for the isolation and cultivation of endophytic fungi are summarized below. $^{13}$

1. Cutting of samples (sponge tissues, mangrove leaves, algal biomass, etc.) into small pieces and removing debris adherent to the surface by rinsing with sterile seawater.

2. Immersing the pieces of sample in $70 \%$ ethanol for surface sterilization. This is to be done carefully to avoid any tissue damage of the sample. To check sterilization effectiveness, it is recommended to take imprints of the tissues on growth medium. Lack of microbial growth will indicate successful sterilization.
3. After drying with a sterile cotton cloth, pieces are streaked over a petri dish containing culture media (such as water agar medium), then again cut into smaller pieces.

4. These pieces are then incubated in media (water agar medium) amended with antibiotics at 20$25^{\circ} \mathrm{C}$ under daylight.

5. After two weeks of incubation, different fungal strains are developed from sample. Hyphal tips of growing fungi are transferred in second media such as potato dextrose agar medium (PDA) for further growth.

6. Isolated pure strains are taxonomiclly identified by observing morphological characteristics, and by DNA amplification and sequencing of the fungal internal transcribed spacer (ITS) region.

\section{Extraction and characterization of endophytic fungal metabolites}

Various fermentation techniques such as shaking or static culture of endophytes at optimized conditions including temperature, light, aeration, etc. are maintained for compound extraction. ${ }^{14,15}$ Fermentation media viz. PDA medium, malt extract medium, liquid Wickerham's medium, etc. are mostly utilized for effective large scale production of bioactive compound. ${ }^{13}$ Separation of mycelia and filtrate is done by filtration followed by extraction of the compound using appropriate organic solvents. Most widely used solvent for this purpose is ethyl acetate. Other solvents used are methanol, dichloromethane, chloroform and hexane, etc. ${ }^{16,19}$

Cell-free organic phase is dried under vacuum at $40^{\circ} \mathrm{C}$ by a rotary evaporator. Fractionation of extracts is done using column chromatography by polarity based gradient elution of the suitable solvent system, and the existence of the compounds is detected by thin layer chromatography (TLC). Further isolation proceeds by preparative TLC, column chromatography, vacuum liquid chromatography or preparative-HPLC, etc. Elucidation of compound structure is carried out using high-resolution nuclear magnetic resonance (NMR) and fourier transform ion cyclotron resonance mass spectrometry (FT-ICR/MS) 
etc. Liquid chromatography-mass spectrometry (LCMS) is also used for compound identification by correlating both molecular weight and UV absorption data. When the individual compounds are purified and structurally recognized, they are then assayed for bioactivity such as cytotoxicity, antimicrobial activity, etc.

\section{Biological potentials of endophytic fungal metabolites}

Metabolic interactions between endophytes and hosts like signaling defense and regulation of symbiosis for adaptation in the highly competitive environment lead to the production of varieties compounds but very little is known about exact biosynthetic pathways behind those. Until now, several hundreds of bioactive metabolites have been identified and isolated from endophytic fungi having a wide range of properties including anticancer, antimicrobial, immunosuppressants activities, etc. (Table 1). World's first billion-dollar anticancer drug taxol shifted natural product research to endophytic fungi initially which could be derived only from some slow-growing rare yew trees. An endophytic fungus, Taxomyces andreanae isolated from yew tree, Taxus brevifolia Nutt. had been reported of having the capability to produce taxol for the first time in 1971 by Wani et al. ${ }^{11}$ In search of other taxol producing endophytes from different other species of yew tree, an endophytic fungus Pestalotiopsis microspora isolated from Taxus wallachiana by Strobel et al. has drawn attention. ${ }^{20}$ Cephalosporin C, another very significant discovery of the era was isolated from marine-derived fungus Acremonium chrysogenum. Till now, more than 1000 novel natural compounds have been discovered by exploring marine-derived fungi haveing new carbon skeleton which indicates marine-associted fungi as a promising source of pharmaceutical lead compounds. Among different genus, Aspergillus and Penicillium are two prolific producers of new metabolites.

\section{Cytotoxic metabolites from marine algicolous endophytic fungi}

\section{Polyketides}

Few dozens of polyketides having cytotoxicity have been isolated from marine algicolous endophytic fungi. A berif of the important endophytes, their sources and isolated cytotoxic metaboliotes are summarized in Table 1. Elsebai et $a l$. found seven novel phenalenone derivatives (1-7) from the endophyte Coniothyrium cereal derived from Enteromorpha sp., a green alga obtained from Fehmarn of Baltic Sea. Among the separated compounds, conioscleroderolide (1) has been showed powerful inhibition of human leukocyte elastase (HLE) having the $\mathrm{IC}_{50}$ value of $13.3 \mu \mathrm{M} .{ }^{36}$ Marine alga Ceramium sp. derived endophytic fungus Phaeosphaeria spartinae was investigated which provide the isolation of a novel compound spartinoxide (9) and two known compounds 4hydroxy-3-prenyl-benzoic acid (10) and anofinic acid (8). Compounds $\mathbf{9}$ and $\mathbf{1 0}$ have been proved to be the potent inhibitor of HLE having $\mathrm{IC}_{50}$ values of $1.71 \pm$ $0.30 \mu \mathrm{g} / \mathrm{ml}(6.5 \mu \mathrm{M})$ and $1.67 \pm 0.32 \mu \mathrm{g} / \mathrm{ml}(8.1 \mu \mathrm{M})$, respectively. ${ }^{37}$ The red alga Carpopeltis cornea (collected in Ulsan City, Korea) derived endophytic fungus Aspergillus parasiticus \# MFA 153 was cultivated which led to the isolation of a novel gabosine derivative parasitenone (11), having moderate antioxidative activity. ${ }^{38}$ Anomalins A (12) and $B(\mathbf{1 3})$, two xanthone derivatives were isolated from a culture of the endophyte Wardomyces anomalus OS4T3-2-1 from the green alga Enteromorpha sp. (collected around Fehmarn island in the Baltic Sea). Anomalin A (12) found active significantly as a p56(lck) tyrosine kinase inhibitor and also as an antioxidant. ${ }^{3}$ Aspergillus pseudodeflectus Hiji005 derived from brown alga Sargassum fusiform (collected in the Miura Peninsula, Japan) produced a new cytotoxic isochroman metabolite, pseudodeflectusin (14). This isolated metabolite showed moderate but selective cytotoxicity against several human cancer cell lines including the stomach (NUGC-3), cervix (HeLa-S3), 
Table 1. Major classes of bioactive compounds derived from marine associated endophytic fungi.

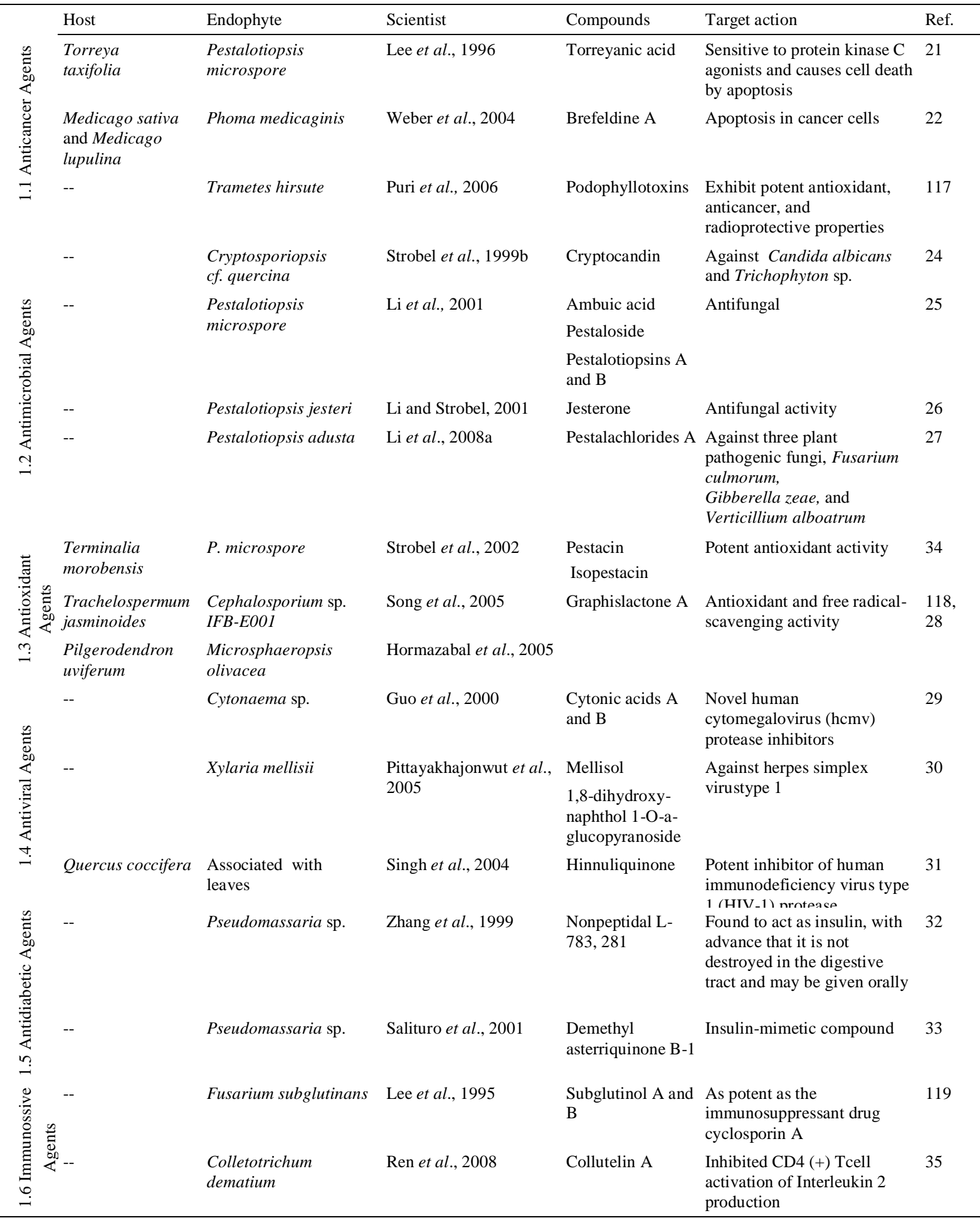

“--" = host not mentioned; Ref. = Reference No. 
and peripheral blood (HL-60), with $\mathrm{LD}_{50}$ values of 49, 47, and $39 \mu \mathrm{M}$, respectively. ${ }^{40}$ A Cladosporium species L037 derived from the brown alga Actinotrichia fragilis (collected from Seragaki Beach at Okinawa Island, Japan) yielded two 12-membered macrolides, sporiolides A (15) and B (16), that showed powerful cytotoxic activity against murine lymphoma L1210 cells. ${ }^{41}$ A known fungal metabolite, 4-Ketoclonostachydiol (17) having potent cytotoxic activity, was obtained from an endophyte Gliocladium sp. isolated from the New Zealand alga Durvillaea Antarctica. ${ }^{42}$ A culture of an endophyte Chaetomium globosum, derived from the red alga Polysiphonia urceolata (collected from Qingdao coastline, China), provided the isolation of a new benzaldehyde secondary metabolite, chaetopyranin (18) which exhibited moderate cytotoxicty against three human tumor cell lines, with $\mathrm{IC}_{50}$ values of 48.7 (human microvascular endothelial cells, HMEC), 90.2 (hepatocellular carcinoma cells, SMMC-7721), and $123.7 \mu \mathrm{M}$ (human lung epithelial cells, A549), along with free radical-scavenging activity. ${ }^{43}$ Same species was isolated that produce a novel benzonaphthyridinedione derivative, chaetominedione (45) having remarkable activity as p56 (lck) tyrosine kinase (93.6\% enzyme inhibition at $200 \mu \mathrm{g} / \mathrm{ml}$ ) inhibitor. ${ }^{44}$ Cultivation of marine green alga-derived endophyte Monodictys putredinis (No. 187/195 15 I, collected from Tenerife, Spain) resulted to the isolation of four monomeric xanthones, including monodictysins A-C (19-21) and monodictyxanthone B (22) as well as a benzophenone monodictyphenone C (23). Among them, monodictysin B (20) showed cytochrome P450 1A inhibition, while monodictysin $\mathrm{C}$ (21) moderately induced $\mathrm{NAD}(\mathrm{P}) \mathrm{H}$ : quinine reductase $(\mathrm{QR})$ activity in mouse Hepa 1c1c7 cells. Also, monodictysin C (21) inhibited aromatase activity weakly. ${ }^{45}$ After fermentation of a culture of a Curvularia sp. (strain no. 768) isolated from the red alga Acanthaphora spicifera (collected in Apra Harbor, Guam), a novel macrolide apralactone A (24), a 14 membered phenyl acetic acid macrolactone, and the antipodes of curvularin macrolides (25-27) were identified. These isolated metabolites showed cytotoxicity against several human tumor cell lines. ${ }^{46}$ Penicillium chrysogenum QEN-24S, obtained from the red alga Laurencia sp. (collected from the Weizhou Island, South China Sea), produced two polyketide penicitides A (28) and B (29) and the glycerol derivative. Penicitide A displayed moderate cytotoxic activity against the human hepatocellular liver carcinoma cell line with an $\mathrm{IC}_{50}$ value of 111.9 $\mu \mathrm{M} .{ }^{47}$ An endophyte Aspergillus terreus, derived from the red alga Laurencia ceylanica (from East coast of Sri Lanka), was a producer of a new butyrolactone (30), which exhibited significant inhibition of the $\beta$ glucuronidase enzyme having $\mathrm{IC}_{50}$ value of $6.2 \mu \mathrm{M}{ }^{48}$ A culture of an endophyte Paecilomyces variotii EN291 obtained from the red alga Grateloupia turuturu (from Qingdao, China) was fermented that led to the isolation of two new butenolides, butyrolactone IX (31) and aspulvinone O (32). Between these, aspulvinone $\mathrm{O}$ was found as a significant antioxidant having $\mathrm{IC}_{50}$ value of $11.6 \mu \mathrm{M} .{ }^{49}$ Halimide alkaloids were produced by Aspergillus sp. CNC139 isolated from a sample of the green alga Halimeda copiosa. Halimide (33) was shown to inhibit the growth of colon cancer and ovarian cancer cell lines with $\mathrm{IC}_{50}$ values of 1 and $0.8 \mu \mathrm{M}$, respectively. ${ }^{50}$ The fungus Emericella nidulans var. acristata was isolated as an endophyte from a Mediterranean green alga. Cultivation of this fungus yielded two new compounds, arugosins $\mathrm{G}(\mathbf{3 4})$ and $\mathrm{H}(\mathbf{3 5})$, together with the known metabolites arugosins $\mathrm{A}(\mathbf{3 6})$ and $\mathrm{B}$ (37). Arugosins A and B exhibited cytotoxic activity towards seven cell lines at the concentration of 10 $\mu \mathrm{M} .{ }^{16}$ Cultivation of endophyte Monodictys putredinis derived from a marine alga resulted to the production of two new dimeric chromanones $(\mathbf{3 8}, \mathbf{3 9})$ consisting two uniquely modified xanthone-derived units. The compounds $(\mathbf{3 8}, 39)$ were examined for their cancer chemopreventive potential and shown to inhibit cytochrome P450 1A activity $\left(\mathrm{IC}_{50}\right.$ values of 5.3 and $7.5 \mu \mathrm{M}$, respectively). In addition, both compounds displayed moderate activity as inducers of $\mathrm{NAD}(\mathrm{P}) \mathrm{H}$ :quinone reductase $(\mathrm{QR})$ in cultured mouse Hepa 1c1c7 cells. ${ }^{51}$ Noduliprevenone (40) 
isolated from Nodulisporium sp. was found to be an inhibitor of P450 (CYP1A), and concomitantly induces $\mathrm{NAD}(\mathrm{P}) \mathrm{H}$ :quinone reductase $(\mathrm{QR}) .{ }^{52} \mathrm{An}$ alga-derived endophytic fungus Penicillium sp. i-1-1 produced a novel tricyclic compound, citrinal A (41) having a rare tetrahydro-2H-benzofuro[7b][1,4]dioxin-9(3H)-one skeleton. Citrinal A exhibited cytotoxicity against the A-549 and HL-60 cell lines. ${ }^{53}$ Cytochalasin D (42), a known antitumor and antibiotic compound was isolated from Xylaria sp. strain obtained from Brazilian marine red alga $B$. tenella. $^{54}$ Epiepoxydon (43) exhibited significant cytotoxicity against human cancer cell lines was obtained from marine fungus Apiospora montagnei isolated from the inner tissue of the North Sea alga Polysiphonia violacea. ${ }^{14}$ An endophyte Ascochyta saliconiae obtained from green alga Ulva sp. produced 2, 3-dihydro-2-hydroxy-2, 4- dimethyl-5trans-propenylfura-3-one (44) showing inhibitory activity towards tyrosine kinase enzyme. ${ }^{55}$ (Table 2, Figure 1)

\section{Terpenoids}

Cytotoxic terpenoids reported so far from marine algicolous endophytes are summarized in Table 3 and Figure 2. Four novel derivatives of hydroxylated sclerosporin were isolated from the fungus Cadophora malorum (SY3-1-1MIT), collected from the green alga Enteromorpha sp. These are 15hydroxysclerosporin (45), 12-hydroxysclerosporin (46),11-hydroxysclerosporin (47), and 8hydroxysclerosporin (48). Among others 8Hydroxysclerosporin was found with a weak lipidaccumulation inhibitory activity against 3T3-L1 murine adipocytes. ${ }^{56}$ Brown alga Sargassum horneri (Wenzhou island, China) derived endophytic fungus Pestalotiopsis sp. Z233 was cultured under abiotic stress stimulated by $\mathrm{CuCl}_{2}$. This culture extracts provided two new stress metabolites, eudesmane sesquiterpenes (49) and (50), which exhibited inhibitory activities against tyrosinase enzyme $\left(\mathrm{IC}_{50}\right.$ values of 14.8 and $22.3 \mu \mathrm{M}$ respectively). ${ }^{57}$ The fungus Penicillium chrysogenum QEN-24S was isolated from the marine red alga Laurencia sp.
Extraction of this fungal culture led to the isolation of two new tetracyclic diterpenes of the rarely reported cyclopiane class, conidiogenones $\mathrm{H}$ and $\mathrm{I}$, along with five related congeners, conidiogenones $\mathrm{B}-\mathrm{D}$ and $\mathrm{F}$ and conidiogenol. Among those conidiogenone $\mathrm{C}$ (51) was found with potent cytotoxic activity against HL-60 and BEL-7402 cells with $\mathrm{IC}_{50}$ values of 0.038 and $0.97 \mu \mathrm{M}$ respectively. ${ }^{58}$ Three new tetranorlabdane diterpenoids, asperolides A-C (5254), and two related tetra norditerpenoid derivatives, wentilactones A and B $(\mathbf{5 5}, \mathbf{5 6})$, were characterized from the culture extracts of Aspergillus wentii EN-48, derived from the marine brown alga Sargassum sp. All of them were evaluated for cytotoxic activity against HeLa, HepG2, MCF-7, MDA-MB-231, NCIH460, SMMC-7721, and SW1990 cell lines with 56 being regarded as the most potent among the tested compounds $\left(\mathrm{IC}_{50}=17 \mu \mathrm{M}\right) .{ }^{59}$ Three new macrocyclic epoxy-diterpenes were produced from an unidentified fungal strain (MPUC 046) derived from the marine brown alga Ishige okamurae. The isolated compounds show similarity to the known platelet activating factor (PAF) antagonists, phomactins, thats why they were named as phomactin I (57), 13-epiphomactin I (58) and phomactin J (59). ${ }^{60}$ (Table 3, Figure 2)

\section{Nitrogenated compounds}

Amines and amides:

Alternaria tenuis Sg17-1 was isolated from an unidentified alga (Zhoushan Island, China) and after the extraction of the culture broth of this fungus, a new isocoumarin (60) containing an atypical sevenmembered ring in its side chain was discovered. Compound 60 exhibited in vitro cytotoxicity against human malignant A375-S2 and Hela cell lines with $\mathrm{IC}_{50}$ values of 0.3 and $0.05 \mathrm{mM}$ respectively. ${ }^{61}$ Methyl 4-(3,4-dihydroxybenzamido) butanoate (61), a novel benzamide derivative was isolated from the endophytic fungus Aspergillus wentii EN-48 derived from marine algae and showed significant DPPH free radical scavenging activity with an $\mathrm{IC}_{50}$ value of 23.1 $\mu \mathrm{M} .{ }^{62}$ Ten asperpyrone-type BNPs (62-71) were characterized from the fungus Aspergillus niger. 
Table 2. Cytotoxic polyketides from marine aglae associated endophytic fungi.

\begin{tabular}{|c|c|c|c|c|c|c|c|}
\hline S1. & Host & Endophytes & Scientist & Compounds & Derivatives & Activities & Ref \\
\hline 1. & Enteromorpha sp. & $\begin{array}{l}\text { Coniothyrium } \\
\text { cereale }\end{array}$ & $\begin{array}{l}\text { Elsebai et al., } \\
2011\end{array}$ & $\begin{array}{l}\text { Conioscleroderolide }(\mathbf{1}), \\
\text { Sclerodine }(\mathbf{2}), \\
\text { Trypethelone }(\mathbf{3}), \\
\text { Coniosclerodin }(\mathbf{4}), \\
\text { Coniosclerolide }(\mathbf{5}), \\
\text { Cereolactam }(\mathbf{6}), \\
\text { Cereoaldomine }(\mathbf{7})\end{array}$ & $\begin{array}{l}\text { Phenalenone } \\
\text { derivatives }\end{array}$ & $\begin{array}{l}\text { Cytotoxic and } \\
\text { antimicrobial activities } \\
(\mathbf{1 - 3}), \\
\text { Inhibitory activity on } \\
\text { HLE (4-7) }\end{array}$ & 36 \\
\hline 2. & Ceramium sp. & $\begin{array}{l}\text { Phaeosphaeria } \\
\text { Spartinae }\end{array}$ & $\begin{array}{l}\text { Elsebai et al., } \\
2010\end{array}$ & $\begin{array}{l}\text { Anofinic acid }(\mathbf{8}), \text { Prenyl } \\
\text { OH benzoic acid }(\mathbf{9}), \\
\text { Spartinoxide }(\mathbf{1 0})\end{array}$ & & $\begin{array}{l}\text { Inhibitory activity on } \\
\text { HLE (8) }\end{array}$ & 37 \\
\hline 3. & $\begin{array}{l}\text { Carpopeltis } \\
\text { cornea red algae }\end{array}$ & $\begin{array}{l}\text { Aspergillus } \\
\text { parasiticus }\end{array}$ & $\begin{array}{l}\text { Son et al., } \\
2002\end{array}$ & Parasitenone (11) & $\begin{array}{l}\text { Gabosine } \\
\text { derivative }\end{array}$ & $\begin{array}{l}\text { Moderate free radical } \\
\text { scavenging activity }\end{array}$ & 38 \\
\hline 4. & Enteromorpha sp. & $\begin{array}{l}\text { Wardomyces } \\
\text { anomalus }\end{array}$ & $\begin{array}{l}\text { Abdel-Lateff } e t \\
\text { al., } 2003\end{array}$ & Anomalin A (12) & $\begin{array}{l}\text { Xanthone } \\
\text { derivatives }\end{array}$ & $\begin{array}{l}\text { Tyrosine kinase } \\
\text { inhibitory \& } \\
\text { antioxidative activity }\end{array}$ & 39 \\
\hline 5. & $\begin{array}{l}\text { Sargassum } \\
\text { fusiform brown } \\
\text { algae }\end{array}$ & $\begin{array}{l}\text { Aspergillus } \\
\text { pseudodeflectus }\end{array}$ & $\begin{array}{l}\text { Ogawa et al., } \\
2004\end{array}$ & Pseudodeflectusin (14) & $\begin{array}{l}\text { Isochroman } \\
\text { derivative }\end{array}$ & $\begin{array}{l}\text { Selective cytotoxic } \\
\text { activity against several } \\
\text { human cancer cell } \\
\text { lines including the } \\
\text { stomach (NUGC-3), } \\
\text { cervix (HeLa-S3), and } \\
\text { peripheral blood (HL } \\
60)\end{array}$ & 40 \\
\hline 6. & $\begin{array}{l}\text { Actinotrichia } \\
\text { fragilis }\end{array}$ & $\begin{array}{l}\text { Cladosporium } \\
\text { L037 species }\end{array}$ & $\begin{array}{l}\text { Shigemori et } \\
\text { al., } 2004\end{array}$ & $\begin{array}{l}\text { Sporiolides A (15) and B } \\
\text { (16) }\end{array}$ & $\begin{array}{l}\text { 12-membered } \\
\text { macrolides }\end{array}$ & $\begin{array}{l}\text { Potent cytotoxicity } \\
\text { against murine } \\
\text { lymphoma L1210 cells }\end{array}$ & 41 \\
\hline 7. & $\begin{array}{l}\text { Durvillaea } \\
\text { Antarctica }\end{array}$ & Gliocladium sp. & $\begin{array}{l}\text { Lang et al., } \\
2006\end{array}$ & $\begin{array}{l}\text { 4-ketoclonostachydiol } \\
\text { (17) }\end{array}$ & Macrodiolide & Strong cytotoxicity & 42 \\
\hline \multirow[t]{2}{*}{8.} & $\begin{array}{l}\text { Polysiphonia } \\
\text { urceolata }\end{array}$ & $\begin{array}{l}\text { Chaetomium } \\
\text { globosum }\end{array}$ & $\begin{array}{l}\text { Wang et al., } \\
2006 \mathrm{~b}\end{array}$ & Chaetopyranin (18) & $\begin{array}{l}\text { Benzaldehyde } \\
\text { derivative }\end{array}$ & $\begin{array}{l}\text { Cytotoxic against } \\
\text { human microvascular } \\
\text { endothelial cells, } \\
\text { hepatocellular } \\
\text { carcinoma cells } \\
\text { (SMMC-7721) and } \\
\text { human lung epithelial } \\
\text { cells }\end{array}$ & 43 \\
\hline & & & $\begin{array}{l}\text { Abdel-Lateff, } \\
2008\end{array}$ & Chaetominedione (45) & $\begin{array}{l}\text { Benzonaphthyr } \\
\text { idinedione } \\
\text { derivative }\end{array}$ & $\begin{array}{l}\text { p56lck tyrosine kinase } \\
\text { inhibitory activity }\end{array}$ & 44 \\
\hline 9. & $\begin{array}{l}\text { Inner tissue of a } \\
\text { marine green alga }\end{array}$ & $\begin{array}{l}\text { Monodictys } \\
\text { putredinis }\end{array}$ & $\begin{array}{l}\text { Krick et al., } \\
2007\end{array}$ & $\begin{array}{l}\text { Monodictysins A-C (19- } \\
\mathbf{2 1}) \text {, } \\
\text { Monodictyxanthone (22) } \\
\text { Monodictyphenone }(\mathbf{2 3})\end{array}$ & $\begin{array}{l}\text { Monomeric } \\
\text { xanthones } \\
\text { Benzophenone }\end{array}$ & $\begin{array}{l}\text { Inhibition of } \\
\text { cytochrome P450 1A } \\
(\mathbf{2 0}), \text { Induction of } \\
\text { NAD(P)H:quinine } \\
\text { reductase }(\mathrm{QR}) \text { in }\end{array}$ & 45 \\
\hline 10. & $\begin{array}{l}\text { Acanthaphora } \\
\text { spicifera }\end{array}$ & Curvularia sp. & $\begin{array}{l}\text { Greve et al., } \\
2008\end{array}$ & $\begin{array}{l}\text { Apralactone A (24), } \\
\text { Antipodes of curvularin } \\
\text { macrolides }(\mathbf{2 5 - 2 7})\end{array}$ & Macrolide & $\begin{array}{l}\text { Cytotoxic towards } \\
\text { human tumor cell lines }\end{array}$ & 46 \\
\hline 11 & Laurencia sp. & $\begin{array}{l}\text { Penicillium } \\
\text { chrysogenum }\end{array}$ & $\begin{array}{l}\text { Gao et al., } \\
2010\end{array}$ & Penicitides A (28) & -- & $\begin{array}{l}\text { Moderate cytotoxic } \\
\text { against the human } \\
\text { hepatocellular liver } \\
\text { carcinoma cell line }\end{array}$ & 47 \\
\hline 12 & $\begin{array}{l}\text { Laurencia } \\
\text { ceylanica }\end{array}$ & $\begin{array}{l}\text { Aspergillus } \\
\text { terreus }\end{array}$ & $\begin{array}{l}\text { Haroon et al., } \\
2013\end{array}$ & New butyrolactone (30) & & $\begin{array}{l}\text { Inhibition of } \beta \text { - } \\
\text { glucuronidase }\end{array}$ & 48 \\
\hline 13 & $\begin{array}{l}\text { Grateloupia } \\
\text { turuturu }\end{array}$ & $\begin{array}{l}\text { Paecilomyces } \\
\text { variotii }\end{array}$ & $\begin{array}{l}\text { Zhang et al., } \\
2015 \mathrm{a}\end{array}$ & $\begin{array}{l}\text { Butyrolactone IX (31), } \\
\text { Aspulvinone O (32) }\end{array}$ & $\begin{array}{l}\text { Butenolide } \\
\text { derivatives }\end{array}$ & $\begin{array}{l}\text { DPPH radical } \\
\text { scavenging activity }(\mathbf{3 2})\end{array}$ & 49 \\
\hline 14 & Halimeda copiosa & Aspergillus sp. & $\begin{array}{l}\text { Fenical et al., } \\
2000\end{array}$ & Halimide (33) & Alkaloid & $\begin{array}{l}\text { Growth inhibition of } \\
\text { colon and ovarian } \\
\text { carcinoma cells }\end{array}$ & 50 \\
\hline
\end{tabular}




\begin{tabular}{|c|c|c|c|c|c|c|c|}
\hline 15 & Unidentified algae & $\begin{array}{l}\text { Emericella } \\
\text { nidulans var. } \\
\text { acristata }\end{array}$ & $\begin{array}{l}\text { Kralj et al., } \\
2006\end{array}$ & $\begin{array}{l}\text { Arugosin G (34), } \\
\text { Arugosin H (35), } \\
\text { Arugosin B (36), } \\
\text { Arugosin A (37) }\end{array}$ & $\begin{array}{l}\text { Prenylated } \\
\text { polyketides }\end{array}$ & $\begin{array}{l}\text { Cytotoxic toward tumor } \\
\text { cell lines }(\mathbf{3 6}, \mathbf{3 7})\end{array}$ & 16 \\
\hline 16 & Unidentified algae & $\begin{array}{l}\text { Monodictys } \\
\text { putredinis }\end{array}$ & $\begin{array}{l}\text { Pontius et al., } \\
2008 \mathrm{~b}\end{array}$ & $\begin{array}{l}\text { Monodictyochromes A } \\
\text { (38) and B (39) }\end{array}$ & $\begin{array}{l}\text { Dimeric } \\
\text { xanthone } \\
\text { derivatives }\end{array}$ & $\begin{array}{l}\text { Inhibit cytochrome } \\
\text { P450 1A activity }\end{array}$ & 52 \\
\hline 17 & Unidentified & $\begin{array}{l}\text { Nodulisporium } \\
\text { sp. }\end{array}$ & $\begin{array}{l}\text { Pontius et al., } \\
\text { 2008a }\end{array}$ & Noduliprevenone (40) & $\begin{array}{l}\text { Heterodimeric } \\
\text { chromanone }\end{array}$ & $\begin{array}{l}\text { Inhibitor of P450 } \\
\text { (CYP1A) }\end{array}$ & 51 \\
\hline 18 & Blidingia minima & Penicillium sp. & $\begin{array}{l}\text { Zhu et al., } \\
2009\end{array}$ & Citrinal A (41) & $\begin{array}{l}\text { Tricyclic } \\
\text { citrinin } \\
\text { derivative }\end{array}$ & $\begin{array}{l}\text { Cytotoxic effects on the } \\
\text { A-549 and HL-60 cell } \\
\text { lines }\end{array}$ & 53 \\
\hline 19 & Bostrychia tenella & Xylaria sp. & $\begin{array}{l}\text { de Felício et } \\
\text { al., } 2015\end{array}$ & Cytochalasin D (42) & Alkaloid & & 54 \\
\hline 20 & $\begin{array}{l}\text { Polysiphonia } \\
\text { violacea }\end{array}$ & $\begin{array}{l}\text { Apiospora } \\
\text { montagnei }\end{array}$ & $\begin{array}{l}\text { Klemke } \text { et al., } \\
2004\end{array}$ & Epiepoxydon (43) & & $\begin{array}{l}\text { Significant cytoxicity } \\
\text { against human cancer } \\
\text { cell lines. }\end{array}$ & 14 \\
\hline 21 & Ulva sp. & $\begin{array}{l}\text { Ascochyta } \\
\text { saliconiae }\end{array}$ & $\begin{array}{l}\text { Nagle et al., } \\
2004\end{array}$ & $\begin{array}{l}\text { 2, 3-dihydro-2-hydroxy- } \\
\text { 2, 4- dimethyl-5-trans- } \\
\text { propenylfura-3-one (44) }\end{array}$ & & $\begin{array}{l}\text { Tyrosine kinase } \\
\text { inhibitor }\end{array}$ & 55 \\
\hline
\end{tabular}

Table 3. Cytotoxic terpenoids from marine aglae associated endophytic fungi.

\begin{tabular}{|c|c|c|c|c|c|c|c|}
\hline S1. & Host & Endophytes & Scientist & Compounds & Derivatives & Activities & $\operatorname{Re}$ \\
\hline 1. & Enteromorpha sp. & $\begin{array}{l}\text { Cadophora } \\
\text { malorum SY3-1- } \\
\text { IMIT }\end{array}$ & $\begin{array}{l}\text { Almeida } \text { et al., } \\
2010\end{array}$ & $\begin{array}{l}\text { 15-hydroxysclerosporin } \\
\text { (45),12-hydroxysclerosporin } \\
\text { (46),11-hydroxysclerosporin } \\
\text { (47),8-hydroxy sclerosporin } \\
\text { (48) }\end{array}$ & $\begin{array}{l}\text { Hydroxylated } \\
\text { sclerosporin } \\
\text { derivatives }\end{array}$ & $\begin{array}{l}\text { Fat-accumulation } \\
\text { inhibitory activity } \\
\text { against 3T3- } \\
\text { L1murine } \\
\text { adipocytes }\end{array}$ & 56 \\
\hline 2. & $\begin{array}{l}\text { Pestalotiopsis sp. } \\
\text { Z233 }\end{array}$ & $\begin{array}{l}\text { Sargassum } \\
\text { horneri }\end{array}$ & $\begin{array}{l}\text { Wu et al., } \\
2013\end{array}$ & 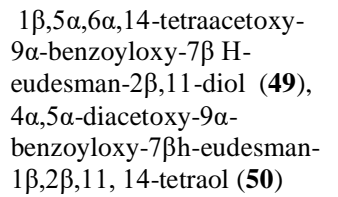 & $\begin{array}{l}\text { Eudesmane } \\
\text { ses- } \\
\text { quiterpenes }\end{array}$ & $\begin{array}{l}\text { Tyrosinase } \\
\text { inhibitory activities }\end{array}$ & 57 \\
\hline 3. & Laurencia sp. & $\begin{array}{l}\text { Penicillium } \\
\text { chrysogenum } Q E \\
N-24 S\end{array}$ & Du et al., 2009 & Conidiogenone C (51) & $\begin{array}{l}\text { Diterpenes of } \\
\text { cyclopiane } \\
\text { class }\end{array}$ & $\begin{array}{l}\text { Cytotoxic effect to } \\
\text { HL-60 }\end{array}$ & 58 \\
\hline 4. & Sargassum sp. & $\begin{array}{l}\text { Aspergillus wentii } \\
\text { EN-48 }\end{array}$ & $\begin{array}{l}\text { Sun } \text { et al., } \\
2012\end{array}$ & $\begin{array}{l}\text { Asperolides A-C (52-54), } \\
\text { Wentilactones A (55) and B } \\
(\mathbf{5 6})\end{array}$ & $\begin{array}{l}\text { Tetranorlabda } \\
\text { ne } \\
\text { diterpenoids }\end{array}$ & $\begin{array}{l}\text { Potent cytotoxic } \\
\text { against a tumor } \\
\text { cells }(\mathbf{5 6})\end{array}$ & 59 \\
\hline 5. & Ishige okamurae & $\begin{array}{l}\text { Unidentified } \\
\text { fungal strain } \\
\text { (MPUC 046) }\end{array}$ & $\begin{array}{l}\text { Ishino et al., } \\
2010\end{array}$ & $\begin{array}{l}\text { Phomactin I (57), 13-epi- } \\
\text { phomactin I (58), Phomactin } \\
\text { J (59) }\end{array}$ & Diterpene & $\begin{array}{l}\text { Platelet activating } \\
\text { factor (PAF) } \\
\text { antagonists }\end{array}$ & 60 \\
\hline 6. & Unidentified alga & Alternaria tenuis & $\begin{array}{l}\text { Huang et al., } \\
2006\end{array}$ & $\operatorname{Sg} 1714(44)$ & $\begin{array}{l}\text { A novel } \\
\text { isocoumarin }\end{array}$ & $\begin{array}{l}\text { Cytotoxicities in } \\
\text { vitro against human }\end{array}$ & 61 \\
\hline 7. & --- & Aspergillus wentii & Li et al., 2014 & $\begin{array}{l}\text { Methyl4-(3,4-dihydroxy } \\
\text { benzamido) butanoate (61) }\end{array}$ & $\begin{array}{l}\text { Benzamide } \\
\text { derivative }\end{array}$ & $\begin{array}{l}\text { malignant A375-S2 } \\
\text { Significant DPPH } \\
\text { radical scavenging }\end{array}$ & 62 \\
\hline 8. & Sargassum sp. & Aspergillus niger & $\begin{array}{l}\text { Fang et al., } \\
2016\end{array}$ & $\begin{array}{l}\text { Aurasperone A (62), } \\
\text { Fonsecinone D }(\mathbf{6 3}), \\
\text { Aurasperone F }(\mathbf{6 4}), \\
\text { Fonsecinone B }(\mathbf{6 5}), \\
\text { Aurasperone B }(\mathbf{6 6}), \\
\text { Aurasperone C }(\mathbf{6 7}), \\
\text { Fonsecinone A }(\mathbf{6 8}), \\
\text { Asperpyrone A (69), } \\
\text { Fonsecinone C (70), } \\
\text { Asperpyrone D (71) }\end{array}$ & & $\begin{array}{l}\text { Weak cytotoxicities } \\
\left(\mathrm{IC}_{50}>30 \mu \mathrm{m}\right) \\
\text { against } 10 \text { human } \\
\text { cancer cell lines } \\
\text { (K562, A549, } \\
\text { Du145, H1975, } \\
\text { MCF-7, Huh-7, } \\
\text { HL7702, HL60, } \\
\text { HeLa, and Molt-4). }\end{array}$ & 63 \\
\hline 9. & Codium fragile. & Gibberella zeae & $\begin{array}{l}\text { Liu et al., } \\
2011\end{array}$ & $\begin{array}{l}\text { 3-hydroxy-5- } \\
\text { (hydroxymethyl)-4-(4'- } \\
\text { hydroxyphenoxy) } \\
\text { pyrrolidin-2-one (72) }\end{array}$ & $\begin{array}{l}\text { Pyrrolidine } \\
\text { derivative }\end{array}$ & $\begin{array}{l}\text { Cytotoxicity against } \\
\text { A-549 and BEL- } \\
7402 \text { cell lines. }\end{array}$ & 64 \\
\hline
\end{tabular}


<smiles>CC(C)CCOC1CC(O)C2C(=O)C(=O)OC3C(O)CC(C)C1C32</smiles>

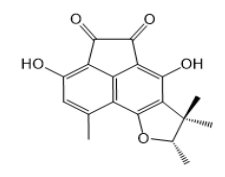

(2)

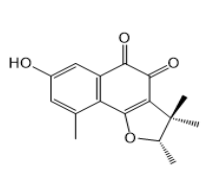

(3)

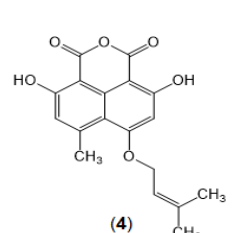

(4) $\mathrm{CH}_{5}$
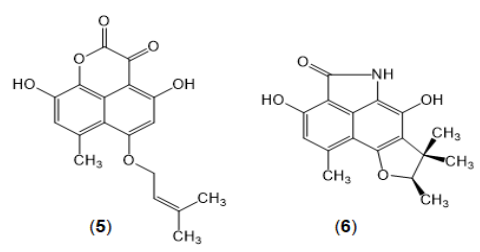

(6)<smiles>CC(C)C=CC1CC(O)CCC1OC(=O)O</smiles><smiles>CC1C(O)C(O)CC2OC3CC(O)C=C(O)C3C(=O)C2C1CO</smiles>

(11) $\mathrm{R}=\mathrm{H} / \mathrm{COCH} 3$<smiles>CC1C(=O)C2OC3CC(O)C=C(O)C3OC2C(O)C1O</smiles><smiles>CC(O)CCCCCCCC(CC(OC(=O)C1CC2CCC1C2)C(=O)O)OC(C)C</smiles><smiles>CC1CCCCCCCCC(=O)CC(O)C(O)C1O</smiles><smiles>CCC(O)CCC(=O)OC(C)C(C)CCC(=O)OC(C)C</smiles><smiles>CC(C)CCC1CCC2C(CC(CCC(C)C)C(O)C2CO)O1</smiles>

(16)

(17) (18)<smiles>CC1CC(O)C2C(=O)C3C(O)C(C)CC(C)C3(C)OC2C1</smiles>

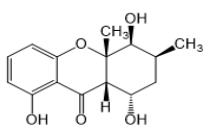

(20)<smiles>COc1cc(O)c2c(c1)OC1(O)C(C(=O)O)C(O)C[C@H](C)[C@H](O)C21O</smiles>

(21)
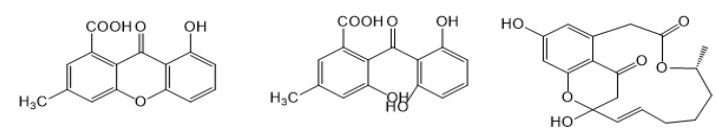

(23)

(24)

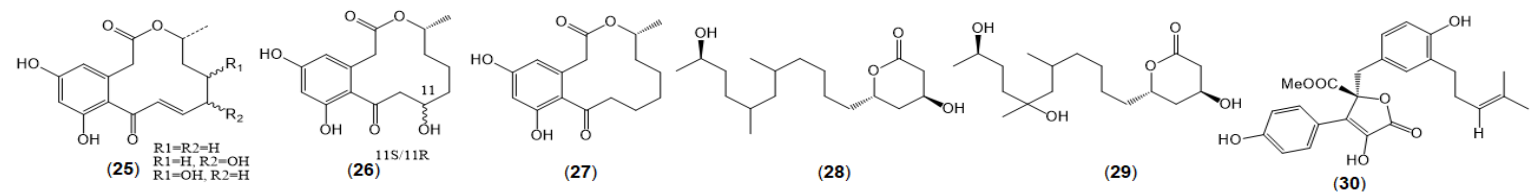<smiles>CC(C)CCCC1CC(C2C(=O)OC(CC3CCC(O)C(CCC(C)C)C3)C2O)C(CCC(C)C)C(O)C1O</smiles>

(31)

(32)

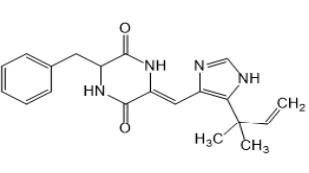

(33)
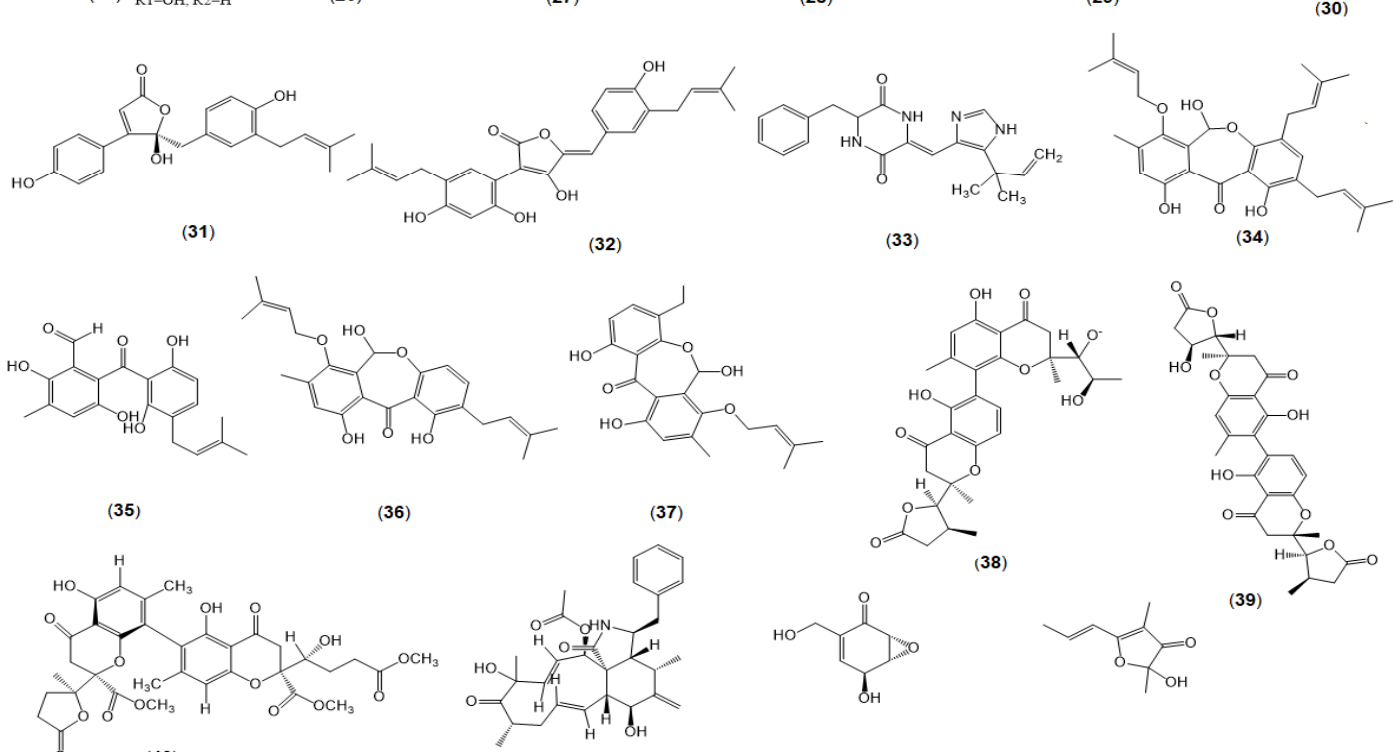

(42)
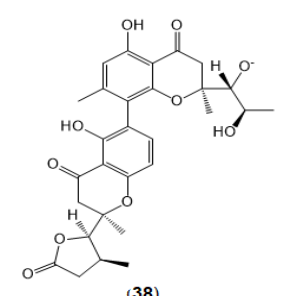

(1)

$(43)$

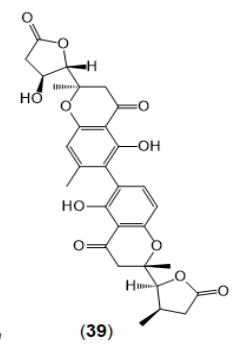

(39)

Figure 1. Cytotoxic polyketidesfrom marine aglae associated endophytic fungi.

SCSIO Jcsw6F30, derived from a marine alga Sargassum sp. All of the BNPs were found as weak cytotoxic compounds against 10 human tumor cells
$\left(\mathrm{IC}_{50}>30 \mu \mathrm{M}\right)$. Besides, three of them, aurasperone $\mathrm{F}$ (62), aurasperone $\mathrm{C}(63)$ and asperpyrone A (64), showed obvious COX-2-inhibitory activities, with 
the $\mathrm{IC}_{50}$ values of $11.1, \quad 4.2$ and $6.4 \mu \mathrm{M}$ respectively. ${ }^{63}$ 3-hydroxy-5-(hydroxymethyl)-4-(4' hydroxyphenoxy) pyrrolidin-2-one (72), a new pyrrolidine derivative was identified from the culture extracts of an endophytic fungus Gibberella zeae, obtained from the marine green alga Codium fragile and showed cytotoxic activity against A-549 and BEL-7402 cell lines. ${ }^{64}$ All the amines and amides with cytotoxic potentials are are summarized in table 4 and the structures are given in figure 3 .

Table 4. Cytotoxic nitrogenated compounds from marine aglae associated endophytic fungi.

\begin{tabular}{|c|c|c|c|c|c|c|c|}
\hline Sl. & Host & Endophytes & Scientist & Compounds & Derivatives & Activities & Ref \\
\hline 1 & $\begin{array}{l}\text { Valonia } \\
\text { utricularis }\end{array}$ & Chaetomium sp. & $\begin{array}{l}\text { Abdel-Lateff, } \\
2008\end{array}$ & Chaetominedione (73) & $\begin{array}{l}\text { Benzonaphthyrid } \\
\text { inedione }\end{array}$ & $\begin{array}{l}\text { Potent tyrosine kinase } \\
\text { inhibitor }\end{array}$ & 44 \\
\hline 2 & $\begin{array}{l}\text { Sargassum } \\
\text { kjellmanianum }\end{array}$ & $\begin{array}{l}\text { Aspergillus } \\
\text { ochraceus }\end{array}$ & $\begin{array}{l}\text { Cui et al., } \\
2009\end{array}$ & $\begin{array}{l}\text { 2-hydroxy } \\
\text { circumdatin C (74) }\end{array}$ & $\begin{array}{l}\text { Benzodiazepine } \\
\text { alkaloids }\end{array}$ & $\begin{array}{l}\text { Exhibited significant } \\
\text { dpph radical scavenging } \\
\text { activity }\end{array}$ & 65 \\
\hline 3 & $\begin{array}{l}\text { Grateloupia } \\
\text { turuturu }\end{array}$ & $\begin{array}{l}\text { Paecilomyces } \\
\text { variotii EN-291 }\end{array}$ & $\begin{array}{l}\text { Zhang et al., } \\
2015 \mathrm{~b}\end{array}$ & $\begin{array}{l}\text { Varioloid A (75) \& B } \\
\text { (76) }\end{array}$ & $\begin{array}{l}\text { Diketopiperazine } \\
\text { type alkaloid }\end{array}$ & $\begin{array}{l}\text { Compounds } 77 \text { and } 78 \\
\text { exhibited cytotoxicity } \\
\text { against A549, HCT } 116 \text {, } \\
\text { and hepg } 2 \text { cell lines, with } \\
\mathrm{IC}_{50} \text { values ranging from } \\
2.6 \text { to } 8.2 \mu \mathrm{g} / \mathrm{ml} \text {. }\end{array}$ & 108 \\
\hline \multirow[t]{2}{*}{4} & $\begin{array}{l}\text { Actinotrichia } \\
\text { fragilis }\end{array}$ & $\begin{array}{l}\text { Penicillium } \\
\text { citrinum N-059 }\end{array}$ & $\begin{array}{l}\text { Tsuda et al., } \\
2004\end{array}$ & Citrinadin A (77) & $\begin{array}{l}\text { Pentacyclic } \\
\text { spiroindolinone } \\
\text { alkaloid }\end{array}$ & $\begin{array}{l}\text { Cytotoxicity against } \\
\text { L1210 and KB cell lines }\end{array}$ & 67 \\
\hline & & & $\begin{array}{l}\text { Mugishima et } \\
\text { al., } 2005\end{array}$ & Citrinadin B (78) & $\begin{array}{l}\text { Pentacyclic } \\
\text { indolinone } \\
\text { alkaloid }\end{array}$ & $\begin{array}{l}\text { Modest cytotoxicity } \\
\text { against } 11210 \text { cells }\end{array}$ & 68 \\
\hline 5 & $\begin{array}{l}\text { Enteromorpha } \\
\text { tubulosa }\end{array}$ & $\begin{array}{l}\text { Aspergillus flavus } \\
\text { C-F-3 }\end{array}$ & $\begin{array}{l}\text { Lin et al., } \\
2009\end{array}$ & $\begin{array}{l}\text { Iso- } \alpha \text {-Cyclo-piazonic } \\
\text { acid (79) }\end{array}$ & & $\begin{array}{l}\text { Cytotoxic to several } \\
\text { human tumor cell lines } \\
\text { (HL-60,MOLT-4,A- } \\
549 \text {,andbel-7402 cell } \\
\text { lines) }\end{array}$ & 69 \\
\hline 6 & Sargassum sp. & $\begin{array}{l}\text { Aspergillus sp. } \\
\text { SpD081030G1f1 }\end{array}$ & $\begin{array}{l}\text { Izumikawa et } \\
\text { al., } 2010\end{array}$ & $\begin{array}{l}\text { JBIR-81 (80) and } \\
\text { JBIR-82 (81) }\end{array}$ & $\begin{array}{l}\text { Terpeptin ana- } \\
\text { logues }\end{array}$ & $\begin{array}{l}\text { Radical scavengers due } \\
\text { to their protective effect } \\
\text { against L-glutamate } \\
\text { toxicity }\end{array}$ & 70 \\
\hline 7 & Ulva pertusa & $\begin{array}{l}\text { Chaetomium } \\
\text { globosum }\end{array}$ & $\begin{array}{l}\text { Cui et al., } \\
2010 \mathrm{a}\end{array}$ & $\begin{array}{l}\text { Cyto-globosins A-G } \\
(\mathbf{8 2 - 8 8})\end{array}$ & Cytochalasans & $\begin{array}{l}\text { Cytotoxic activity against } \\
\text { the a- } 549 \text { tumor cell line }\end{array}$ & 71 \\
\hline 8 & $\begin{array}{l}\text { Enteromorpha } \\
\text { sp. }\end{array}$ & $\begin{array}{l}\text { Coniothyrium } \\
\text { cereal }\end{array}$ & $\begin{array}{l}\text { Elsebai } \text { et al., } \\
2012\end{array}$ & Conioimide (89) & $\begin{array}{l}\text { Isoindole } \\
\text { pseudoalkaloid }\end{array}$ & $\begin{array}{l}\text { Selective inhibition of } \\
\text { human leukocyte elastase }\end{array}$ & 72 \\
\hline 9 & $\begin{array}{l}\text { Sargassum } \\
\text { tortile }\end{array}$ & Leptosphaeria sp. & $\begin{array}{l}\text { Takahashi et } \\
\text { al., } 1994\end{array}$ & Lep F (91) and C (92) & Leptosin & $\begin{array}{l}\text { Strong growth-inhibiting } \\
\text { and apoptosis-inducing } \\
\text { activities against human } \\
\text { lymphoblastoid } \\
\text { RPMI8402 cells and } \\
\text { human embryonic kidney } \\
\text { cell line } 293 \text { cells }\end{array}$ & 73 \\
\hline 10 & Avrainvillea sp. & $\begin{array}{l}\text { Fusarium sp. CNL- } \\
619\end{array}$ & $\begin{array}{l}\text { Cueto et al., } \\
2000\end{array}$ & $\begin{array}{l}\text { N-methyl- } \\
\text { sansalvamide (93) }\end{array}$ & $\begin{array}{l}\text { Cyclic } \\
\text { depsipeptide }\end{array}$ & $\begin{array}{l}\text { Weak in vitro } \\
\text { cytotoxicity in the NCI } \\
\text { human tumor cell line }\end{array}$ & 75 \\
\hline 10. & $\begin{array}{l}\text { Lomentaria } \\
\text { catenate }\end{array}$ & Aspergillus sp. & Li et al., 2004 & Golmaenone (94) & $\begin{array}{l}\text { Diketopiperazine } \\
\text { alkaloid }\end{array}$ & $\begin{array}{l}\text { Significant radical } \\
\text { scavenging and UV-A } \\
\text { protecting properties }\end{array}$ & 76 \\
\hline 11. & $\begin{array}{l}\text { Ceradiction } \\
\text { spongiosum }\end{array}$ & Unidentified & $\begin{array}{l}\text { Komatsu et al., } \\
2001\end{array}$ & Dictyonamides A (95) & Peptides & $\begin{array}{l}\text { Inhibited cyclin- } \\
\text { dependent kinase } 4\end{array}$ & 77 \\
\hline
\end{tabular}


Table 5. Cytotoxic steroids from marine aglae associated endophytic fungi.

\begin{tabular}{|c|c|c|c|c|c|c|c|}
\hline Sl. & Host & Endophytes & Scientist & Compounds & Derivatives & Activities & Ref \\
\hline 1. & $\begin{array}{l}\text { Phaeosphaeria } \\
\text { spartinae }\end{array}$ & Ceramium sp. & $\begin{array}{l}\text { Elsebai } \text { et al., } \\
2013\end{array}$ & $\begin{array}{l}\text { Spartopregnenolone } \\
\text { (97) }\end{array}$ & $\begin{array}{l}4 \alpha \text {-carboxylic } \\
\text { pregnane- } \\
\text { derivative }\end{array}$ & Steroidal & 78 \\
\hline 2. & $\begin{array}{l}\text { Sargassum } \\
\text { kjellmanianum }\end{array}$ & $\begin{array}{l}\text { Aspergillus } \\
\text { ochraceus EN-31 }\end{array}$ & $\begin{array}{l}\text { Cui et al., } \\
\text { 2010b }\end{array}$ & $\begin{array}{l}\text { 7-Nor-ergosterolide, } \\
\text { (98) } \\
\text { 3ß-hydroxyergosta-8, } \\
\text { 4(28)-dien-7-one (99) }\end{array}$ & 7-norsteroid & $\begin{array}{l}\text { Cytotoxicity against } \\
\text { NCI-H460,SMMC- } \\
7721 \text {, and SW } 1990 \\
\text { cell lines }\end{array}$ & 79 \\
\hline 3. & $\begin{array}{l}\text { Corallina } \\
\text { officinalis }\end{array}$ & $\begin{array}{l}\text { Aspergillus flavus } \\
\text { Cf-5 }\end{array}$ & $\begin{array}{l}\text { Qiao et al., } \\
2011\end{array}$ & $\begin{array}{l}\text { 3 } \beta, 4 \alpha \text {-dihydroxy-26- } \\
\text { methoxyergosta- } \\
\text { 7,24(28)-dien-6-one }\end{array}$ & & $\begin{array}{l}\text { Low inhibition of } \\
\text { acetylcholinesterase } \\
\text { (AChE) }\end{array}$ & 18 \\
\hline
\end{tabular}

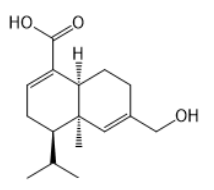

(45)

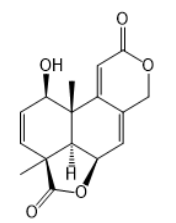

(52)

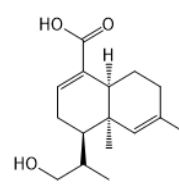

(46)

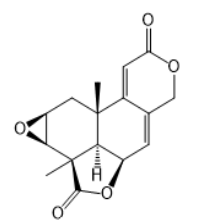

(53)

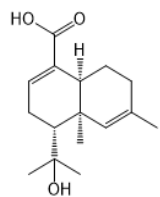

(47)
(48)

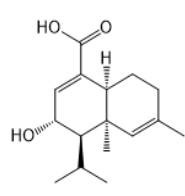

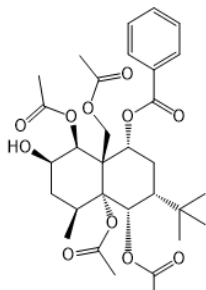

(49)
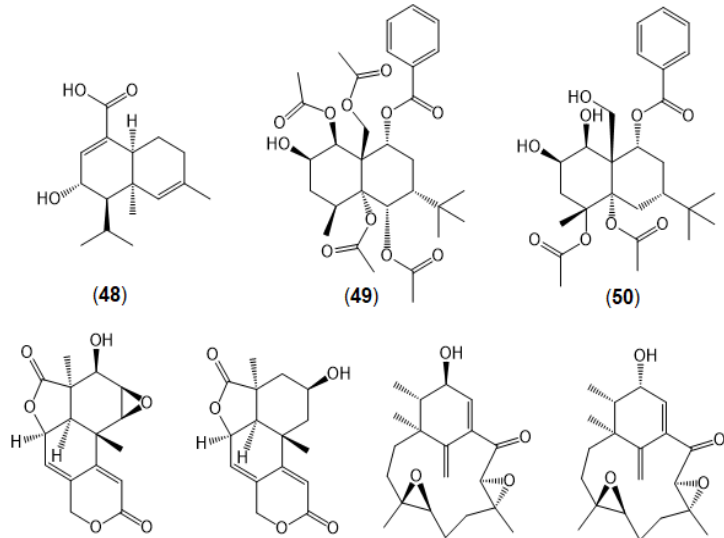

(55)

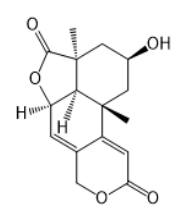

(56)

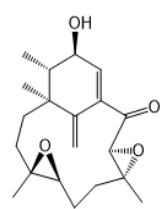

(57)
$(50)$

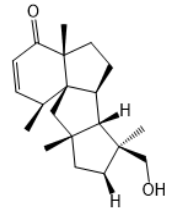

(51)

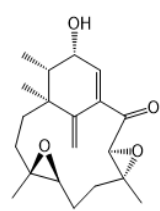

(58)

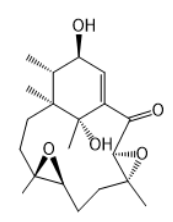

(59)

Figure 2. Cytotoxic terpenoids from marine algae associated endophytic fungi.

\section{Quinolines and quinazoline derivatives:}

From a culture of Chaetomium sp. Az 3-10 obtained from the marine alga Valonia utricularis (Azores, Atlantic Ocean) chaetominedione (73), a new benzonaphthyridinedione derivative was characterized which showed significant tyrosine kinase inhibitory activity. ${ }^{64}$ The endophytic fungus Aspergillus ochraceus, obtained from the marine brown alga Sargassum kjellmanianum produced a novel benzodiazepine analogue, 2hydroxycircumdatin $\mathrm{C}$ (74) in its culture. The culture extracts also provide another compound (11aS)-2,3dihydro-7-methoxy-1H-pyrrolo[2,1 c] [1,4] benzodiazepine-5,11(10H,11aH)-dione, which has been identified from a natural resource for the first time, but has been synthesized previously, along with five structurally related known alkaloids. Compound 74 showed potent DPPH free radical scavenging activity and an $\mathrm{IC}_{50}$ value of $9.9 \mu \mathrm{M}$, which is 8.9fold more potent than that of butylated hydroxytoluene (BHT), a well-known synthetic positive control. ${ }^{65}$ Varioloid A (75), a new indolyl6,10b-dihydro-5a $H$-[1]benzofuro[2,3- $b$ ]indole derivative, and another similar compound varioloid $\mathrm{B}$ (76), was characterized from the endophytic fungus Paecilomyces variotii EN-291 derived from an unidentified marine alga ${ }^{66}$ (Table 4, Figure 3 )

\section{Indole derivatives:}

Citrinadin A (77), a novel pentacyclic spiroindolinone alkaloid, was isolated from the endophytic fungus Penicillium citrinum N-059, derived from marine red alga Actinotrichia fragilis (Hedo Cape, Okinawa Island) and showed moderate cytotoxic activity against L1210 and KB cell lines (with $\mathrm{IC}_{50}$ values of 9.9 and $16.0 \mu \mathrm{M}$ respectively). ${ }^{67}$ The same fungus also provided a new congener, citrinadin B (78), having moderate cytotoxicity against $\mathrm{L} 1210$ cells (with an $\mathrm{IC}_{50}$ value of 20.8 $\mu \mathrm{M}){ }^{68}$ The fungus Aspergillus flavus C-F-3, obtained 


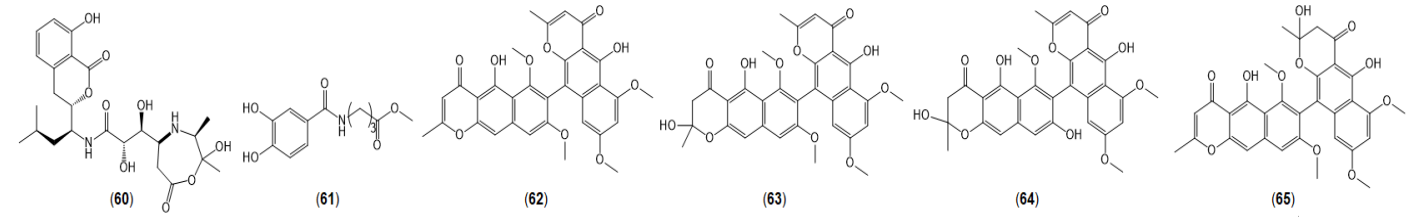
(n)

(66)

(67)

(68)

(69)

(70)

(71)
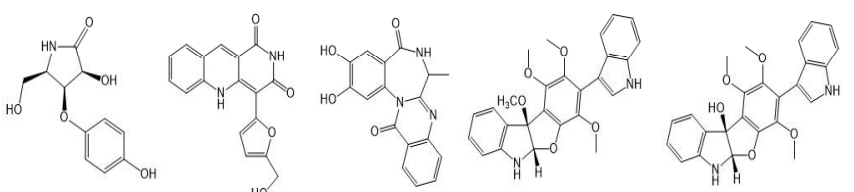

(76)

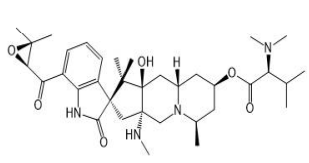

(77)

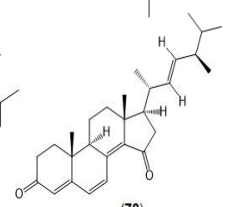

(79)

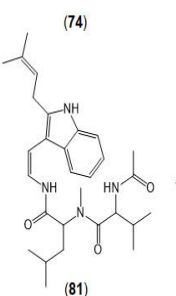

(75)

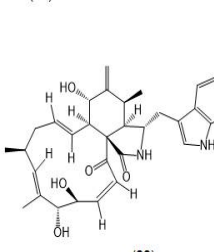

(82)

(83)
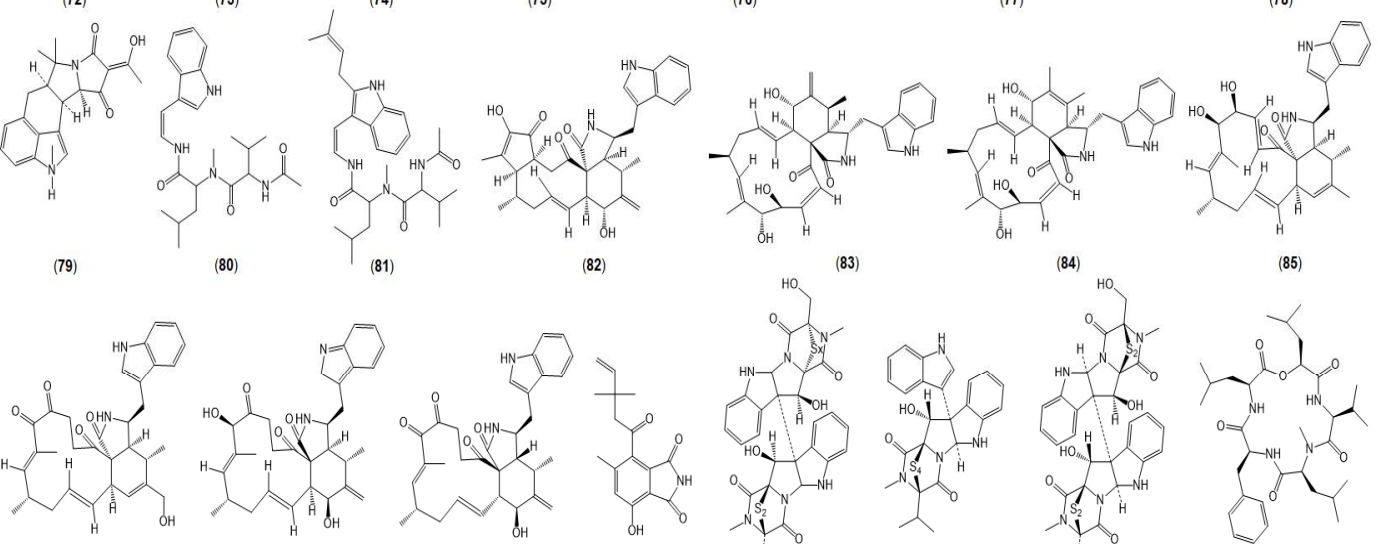

(80)
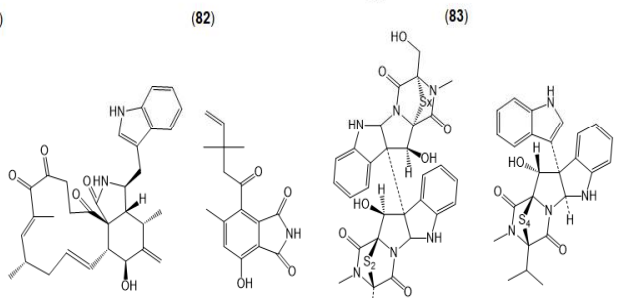

(84)

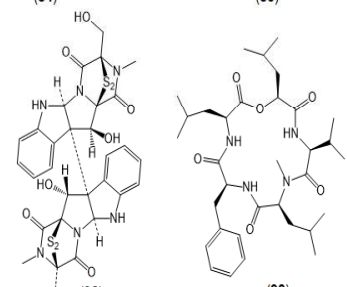

(86)

(87)

(88)

(8)

${ }^{0}(90)$

(91)

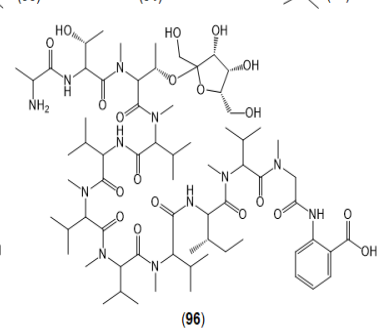

Figure 3. Cytotoxic nitrogenated compounds from marine aglae associated endophytic fungi.

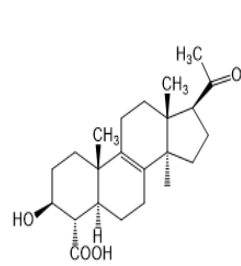

(97)

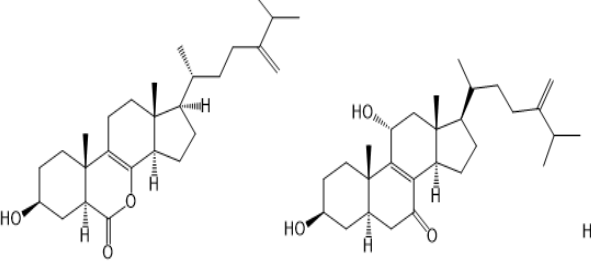

(98)

(99)

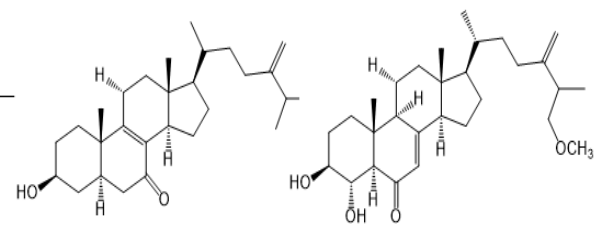

(101)

Figure 4. Cytotoxic steroids from marine algae associated endophytic fungi. 


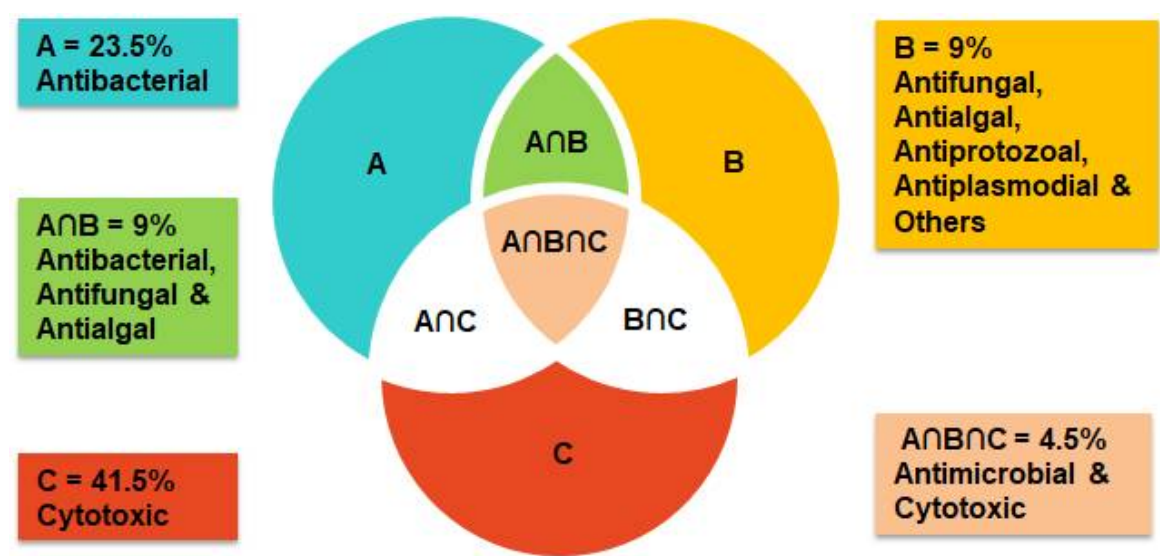

Figure 5. Different classes of bioactive compounds isolated from marine algicolous endophytic fungi upto 2017 (No compound has fallen into $\mathrm{A} \cap \mathrm{C} \& \mathrm{~B} \cap \mathrm{C}$ groups).

from green alga Enteromorpha tubulosa (Putian Pinghai, China), is the source of a moderate cytotoxic compound iso- $\alpha$-cyclopiazonic acid (79), having activity against several human tumor cell lines (HL 60, MOLT- 4, A-549, and BEL-7402 cell lines). ${ }^{69}$ Two novel fungus-derived terpeptin analogues, namely JBIR-81 (80) and JBIR-82 (81), were found as potent free radical scavengers because of their inhibitory activities against L-glutamate toxicity (with $\mathrm{EC}_{50}$ values of 0.7 and $1.5 \mu \mathrm{M}$ ), were isolated from an Aspergillus sp. SpD081030G1f1 obtained from the brown alga Sargassum sp. (Ishigaki Island, Okinawa Prefecture, Japan $)^{70}$. Seven novel cytochalasan derivatives were isolated from the culture of an endophytic fungus Chaetomium globosum QEN-14, obtained from the marine green alga Ulva pertusa (Qingdao coastline, China), which were named as Cytoglobosins A-G (82-88). Among those, cytoglobosins $\mathrm{C}$ and $\mathrm{D}$ showed cytotoxicity against the A-549 tumor cell line ( $\mathrm{IC}_{50}$ values of 2.26 and $2.55 \mu \mathrm{M}$ respectively). ${ }^{71}$ The endophyte Coniothyrium cereale of the green alga Enteromorpha sp. provided the isoindole pseudoalkaloid conioimide (89) from its culture. The compound 89 showed selective inhibitory activity against human leukocyte elastase. ${ }^{72}$ Epipolysulfanyldioxopiperazines (90), a member of the family of leptosins, was produced from the cultured mycelia of the endophytic fungus Leptosphaeria sp. obtained from the brown alga Sargasssum tortile. Leptosin A
(90) showed cytotoxic activity against P-388 leukemia cells through inhibition of topoisomerase II. $^{73}$ Leps $\mathrm{F}$ and $\mathrm{C}(\mathbf{9 1}, \mathbf{9 2})$ were also found from same fungal species. These isolated metabolites exhibited significant growth-inhibition and apoptosisinduction of human lymphoblastoid RPMI8402 cells and human embryonic kidney cell line 293 cells. Furthermore, leps $\mathrm{F}$ and $\mathrm{C}$ also have inhibitory activity in the survival pathway by inactivating (i.e. through dephosphorylation) Akt/protein kinase B enzyme. $^{74}$ The sources of cytotoxic endophytes and the structures of some important indole derivatives are summarized in Table 4 and Figure 3, respectively.

\section{Peptides:}

Few peptides with cytotoxic activities from marine associated algicolous endophytes are also reported (Table 4, Figure 3). A novel cyclic depsipeptide, N-methylsansalvamide (93), was isolated from the culture of an endophyte Fusarium sp. CNL-619, derived from the green alga Avrainvillea sp. (Bovoni Cay, United States Virgin Islands). The compound $\mathbf{9 3}$ showed weak cytotoxic activity against NCI human tumor cell line with a $\mathrm{GI}_{50}$ value of $8.3 \mu \mathrm{M}^{75}$ Golmaenone (94), a diketopiperazine alkaloid, was found as a potent free radical scavenger (with an $\mathrm{IC}_{50}$ value of $20 \mu \mathrm{M}$ ) and UV-A protector (with an $\mathrm{ED}_{50}$ value of $90 \mu \mathrm{M}$ ) from the culture extracts of an Aspergillus species (\# MFA 212) obtained from a red alga Lomentaria catenata 
(Golmae Village, Ulsan City, Korea). ${ }^{76}$ Investigations of an unknown fungus separated from the red alga Ceradictyon spongiosum (Seragaki Beach, Okinawa) led to the identification of two metabolites, dictyonamides A (95) and B (96) of linear dodecapeptide class. Dictyonamide A showed inhibitory activity against cyclin-dependent kinase 4 enzyme (with an $\mathrm{IC}_{50}$ value of $13.0 \mu \mathrm{M}$ ), whereas dictyonamide $\mathrm{B}$ showed inactivity $\left(\mathrm{IC}_{50}\right.$ value $>30$ $\mu \mathrm{M}){ }^{77}$

\section{Steroids}

A compound named spartopregnenolone (97) was isolated from an endophytic fungus Phaeosphaeria spartinae of the marine red alga Ceramium sp. having cytotoxic activity. ${ }^{78}$ 7-Norergosterolide (98), a rare 7-norsteroid with an uncommon pentalactone B-ring system, along with two related derivatives, $3 \beta, 11 \alpha$-dihydroxyergosta8,24(28)-dien-7-one (99) and $3 \beta$ hydroxyergosta8,24(28)-dien-7-one (100), were isolated from the endophyte Aspergillus ochraceus EN-31, obtained from the marine brown alga Sargassum kjellmanianum (Dalian coastline, China). Compounds 98 and 100 were found for the first time from an Aspergillus sp., however, these were previously reported from a Penicillium sp. The compound $\mathbf{9 8}$ showed cytotoxic activity against NCI-H460, SMMC-7721 and SW1990 cell lines with $\mathrm{IC}_{50}$ values of $12.1, \quad 16.9$ and $67.6 \mu \mathrm{M}$ respectively. ${ }^{79}$ Investigation of the endophyte Aspergillus flavus cf-5 of the marine red alga Corallina officinalis (Yantai, China), led to the isolation of a novel steroid (101) from its culture extracts. Compound 101 showed mild inhibitory activity against acetylcholinesterase (AChE) enzyme. ${ }^{18}$ The sources of endophytes and the structures of steroids isolated from them are given in Table 5 and Figure 4, respectively.

\section{CONCLUSION}

Algicolous fungi studied up until now produce both structurally and functionally diverse compounds including steroids, alkaloids, tannins, terpenoids, phenolic acids, quinines, xanthones exhibiting anticancer, antimicrobial, antidiabetic, antioxidant, immunosuppressant and many more properties. In this review we have summarized and explored the possibility of marine algicolous endophytic fungi as a potential source of cytotoxic compounds. Venn diagram analysis (Figure 5) on the isolated compounds, showed that among all the reported cytotoxic and antimicrobial metabolites $41.5 \%$ metabolited were reported to have cytotoxic properties, $23.5 \%$ metabolites were reported to have antibacterial activities. In common, only $9 \%$ of the isolated metabolites showed antibacterial, antifungal and antialgal activities together, while other $9 \%$ were reported to have antifungal, antialgal, antiprotozoal, antiplasmodial and other activities. Among the reported metabolites in this review only $4.5 \%$ have both antimicrobial and cytotoxic effects. This analasys further indicated the importance of marine algicolous endophytic fungi as promising source for future anticancer drug discovery. However, most of this treasure is yet to be discovered. Until now, there is also lack of effective and efficient techniques to isolate potential strains. On the other hand, we often consider only the faster growing culturable strain of endophyties, but ignore the slow growing and unculturable strains. This might be a flaw in the endophytic research because such slow growing strains might have potential to produce high value metabolites. Hence, optimum fermentation conditions including media selection, different culture conditions, etc. are required to adjust considering the physiological and genetic scenario of endophytes. This might stimulate tranquil gene clusters responsible for secondary metabolism. Though many metabolites of endophytic fungi showed prospective bioactivities, but some of them were found inactive or showed weak activities in current reports. This was probably due to bias in the screening program. So, more effective and sensitive high-throughput screening models are strongly recommended for the search of potent cytotoxic metabolites. Particularly computer-aided virtual screening models can play a great role in exploring leads. 


\section{REFERENCES}

1. Tan, R.X. and Zou, W.X. 2001. Endophytes: a rich source of functional metabolites. Nat. Prod. Rep. 18, 448-459.

2. Stone, J.K., Bacon C.W. and White, J.F. 2000. An overview of endophytic microbes: endophytism defined. In: Microbial endophytes (Bacon, C.W. and White, J.F., Eds.), Dekker, New York, pp. 330.

3. Schulz, B. and Boyle, C. 2006. What are endophytes? In: Microbial root endophytes (Schulz, B., Boyle, C. and Sieber, T.N., Eds.), Springer, Berlin, pp. 113.

4. Rodriguez, R.J., White, J.F., Arnold, A.E. and Redman, R.S. 2009. Fungal endophytes: diversity and functional roles. New Phytol. 182, 314-330. doi:10.1111/j.14698137.2009.02773.x

5. Strobel, G.A. 2002. Rainforest endophytes and bioactive products. Crit. Rev. Biotechnol. 22, 315-333. doi:10.1080/07388550290789531

6. Arnold, A.E., Maynard, Z., Gilbert, G.S., Coley, P.D. and Kursar, T.A. 2000. Are tropical fungal endophytes hyperdiverse? Ecol. Lett. 3, 267-274.

7. Schulz, B., Boyle, C. and Draeger, S. 2002. Endophytic fungi: A source of biologically active secondary metabolites. Mycol. Res. 106, 996-1004.

8. Hollants, J., Leliaert, F., De Clerck, O. and Willems, A. 2010. How endo is endo?: surface sterilization of delicate samples: a Bryopsis (Bryopsidales, Chlorophyta) case study. Symbiosis. 51, 131-138.

9. Strobel, G. and Daisy, B. 2003. Bioprospecting for microbial endophytes and their natural products. Microbiol. Mol. Biol. Rev. 67, 491-502. doi:10.1128/MMBR.67.4.491502.2003

10. Strobel, G.A. 2003. Endophytes as sources of bioactive products. Microbes Infect. 5, 535-544.

11. Strobel, G., Yang, X., Sears, J., Kramer, R., Sidhu, R.S. and Hess, W.M. 1996. Taxol from Pestalotiopsis microspora, an endophytic fungus of Taxus wallachiana. Microbiology. (Reading, Engl.) 142 (Pt 2), 435-440.

12. Bacon, C.W. and White, J.F. 1994. Stain, media and procedure for analyzing endophytes. In: Biotechnology of endophytic fungi of grasses (Bacon, C.W. and White, J.F., Eds.), CRC Press, Boca Raton, pp. 47-56.

13. Kjer, J., Debbab, A., Aly, A.H. and Proksch, P. 2010a. Methods for isolation of marine derived endophytic fungi and their bioactive secondary products. Nat. Protoc. 5, 479490. doi:10.1038/nprot.2009.233

14. Klemke, C., Kehraus, S., Wright, A.D. and König, G.M. 2004. New secondary metabolites from the marine endophytic fungus Apiospora montagnei. J. Nat. Prod. 67, 1058-1063. doi:10.1021/np034061x

15. Schulz, B., Draeger, S., Dela, C.T.E., Rheinheimer, J., Siems, K., Loesgen, S., Bitzer, J., Schloerke, O., Zeeck, A., Kock, I., Hussain, H., Dai, J. and Krohn, K. 2008. Screening strategies for obtaining novel, biologically active, fungal secondary metabolites from marine habitats. Botanica Marina. 51, 219234. doi:10.1515/BOT.2008.029
16. Kralj, A., Kehraus, S., Krick, A., Eguereva, E., Kelter, G., Maurer, M., Wortmann, A., Fiebig, H.H. and König, G.M. 2006. Arugosins $\mathrm{G}$ and $\mathrm{H}$ : prenylated polyketides from the marine derived fungus Emericella nidulans var. acristata. J. Nat. Prod. 69, 995-1000. doi:10.1021/np050454f

17. Naganuma, M., Nishida, M., Kuramochi, K., Sugawara, F., Yoshida, H. and Mizushina, Y. 2008. 1-deoxyrubralactone, a novel specific inhibitor of families $\mathrm{X}$ and $\mathrm{Y}$ of eukaryotic DNA polymerases from a fungal strain derived from sea algae. Bioorg. Med. Chem. 16, 2939-2944. doi:10.1016/j.bmc.2007.12.044

18. Qiao, M.F., Ji, N.Y., Miao, F.P. and Yin, X.L. 2011. Steroids and an oxylipin from an algicolous isolate of Aspergillus flavus. Magn. Reson. Chem. 49, 366-369.

19. Erbert, C., Lopes, A.A., Yokoya, N.S., Furtado, N.A.J.C., Conti, R., Pupo, M.T., Lopes, J.L.C. and Debonsi, H.M. 2012. Antibacterial compound from the endophytic fungus Phomopsis longicolla isolated from the tropical red seaweed Bostrychia radicans. Botanica Marina. 55, 435-440. doi:10.1515/bot20110023

20. Strobel, G., Stierle, A., Stierle D. and Hess, W.M. 1993. Taxomyces andreanaea proposed new taxon for a bulbilliferous hyphomycete associated with Pacific yew. Mycotaxon. 47: 71-78.

21. Lee, J.C., Strobel, G.A., Lobkovsky, E. and Clardy, J. 1996. Torreyanic acid: a selectively cytotoxic quinone dimer from the endophytic fungus Pestalotiopsis microspora. J. Org. Chem. 61, 3232-3233. doi:10.1021/jo960471x

22. Weber, R.W.S., Stenger, E., Meffert, A. and Hahn, M. 2004. Brefeldin: a production by Phoma medicaginis in dead precolonized plant tissue: a strategy for habitat conquest? Mycol. Res. 108, 662-671.

23. Korkina, L.G. 2007. Phenylpropanoids as naturally occurring antioxidants: from plant defense to human health. Cell. Mol. Biol. (Noisylegrand) 53, 15-25.

24. Strobel, G., Miller, R.V., Miller, C., Condron, M., Teplow, D.B. and Hess WM. 1999b. Cryptocandin, a potent antimycotic from the endophytic fungus Cryptosporiopsis cf. quercina. Microbiology. 145, 1919-1926.

25. Li, J.Y., Harper, J.K., Grant, D.M., Tombe, B.O., Bashyal, B., Hess, W.M. and Strobel, G.A. 2001. Ambuic acid, a highly functionalized cyclohexenone with antifungal activity from Pestalotiopsis sp. and Monochaetia sp. Phytochemistry. 56, 463-468.

26. Li, J.Y. and Strobel, G.A. 2001. Jesterone and hydroxyjesteroneantioomycete cyclohexenone epoxides from the endophytic fungus Pestalotiopsis jesteri. Phytochemistry. 57, 261-265

27. Li, E., Jiang, L., Guo, L., Zhang, H. and Che, Y. 2008a. Pestalachlorides AC, antifungal metabolites from the plant endophytic fungus Pestalotiopsis adusta. Bioorg. Med. Chem. 16, 7894-7899.

28. Hormazabal, E., Astudillo, L., Schmeda Hirschmann, G., Rodríguez, J. and Theoduloz, C. 2005. Metabolites from Microsphaeropsis olivacea, an endophytic fungus of Pilgerodendron uviferum. Z. Naturforsch. 60, 11-21. doi: $10.1515 /$ znc20051203 
29. Guo, B., Dai, J.R., Ng, S., Huang, Y., Leong, C., Ong, W. and Carté, B.K. 2000. Cytonic acids A and B: novel tridepside inhibitors of hCMV protease from the endophytic fungus Cytonaema species. J. Nat. Prod. 63, 602-604.

30. Pittayakhajonwut, P., Suvannakad, R., Thienhirun, S., Prabpai, S., Kongsaeree, P. and Tanticharoen, M. 2005. An anti-herpes simplex virustype 1 agent from Xylaria mellisii (BCC 1005). Tetrahedron Lett. 46, 1341-1344. doi:10.1016/j.tetlet.2004.12.110

31. Singh, S.B., Ondeyka, J.G., Tsipouras, N., Ruby, C., Sardana, V., Schulman, M., Sanchez, M., Pelaez, F., Stahlhut, M.W., Munshi, S., Olsen, D.B. and Lingham, R.B. 2004. Hinnuliquinone, a C-2symmetric dimeric nonpeptide fungal metabolite inhibitor of HIV1 protease. Biochem. Biophys. Res. Commun. 324, 108-113. doi:10.1016/j.bbrc.2004.08.234

32. Zhang, B., Salituro, G., Szalkowski, D., Li, Z., Zhang, Y., Royo, I., Vilella, D., Díez, M.T., Pelaez, F., Ruby, C., Kendall, R.L., Mao, X., Griffin, P., Calaycay, J., Zierath, J.R., Heck, J.V., Smith, R.G. and Moller, D.E. 1999. Discovery of a small molecule insulin mimetic with antidiabetic activity in mice. Science. 284, 974-977.

33. Salituro, G.M., Pelaez, F. and Zhang, B.B. 2001. Discovery of a small molecule insulin receptor activator. Recent Prog. Horm. Res. 56, 107-126.

34. Strobel, G., Ford, E., Worapong, J., Harper, J.K., Arif, A.M., Grant, D.M., Fung, P.C.W. and Ming Wah Chau, R. 2002. Isopestacin, an isobenzofuranone from Pestalotiopsis microspora, possessing antifungal and antioxidant activities. Phytochemistry. 60, 179-183.

35. Ren, Y., Strobel, G.A., Graff, J.C., Jutila, M., Park, S.G., Gosh, S., Teplow, D., Condron, M., Pang, E., Hess, W.M. and Moore, E. 2008. Colutellin A, an immunosuppressive peptide from Colletotrichum dematium. Microbiology. (Reading, Engl.) 154, 1973-1979.

36. Elsebai, M.F., Kehraus, S., Lindequist, U., Sasse, F., Shaaban, S., Gütschow, M., Josten, M., Sahl, H.G. and König, G.M. 2011. Antimicrobial phenalenone derivatives from the marine derived fungus Coniothyrium cereale. Org. Biomol. Chem. 9, 802-808. doi:10.1039/c0ob00625d

37. Elsebai, M.F., Kehraus, S., Gütschow, M. and König, G.M. 2010. Spartinoxide, a new enantiomer of A82775C with inhibitory activity toward HLE from the marine derived fungus Phaeosphaeria spartinae. Nat. Prod. Commun. 5, 1071-1076.

38. Son, B.W., Choi, J.S., Kim, J.C., Nam, K.W., Kim, D.S., Chung, H.Y., Kang, J.S. and Choi, H.D. 2002. Parasitenone, a new epoxycyclohexenone related to gabosine from the marine derived fungus Aspergillus parasiticus. J. Nat. Prod. 65, 794-795.

39. Abdel-Lateff, A., Klemke, C., König, G.M. and Wright, A.D. 2003. Two new xanthone derivatives from the algicolous marine fungus Wardomyces anomalus. J. Nat. Prod. 66, 706708. doi:10.1021/np020518b
40. Ogawa, A., Murakami, C., Kamisuki, S., Kuriyama, I., Yoshida, H., Sugawara, F. and Mizushina, Y. 2004. Pseudodeflectusin, a novel isochroman derivative from Aspergillus pseudodeflectus a parasite of the sea weed, Sargassum fusiform, as a selective human cancer cytotoxin. Bioorg. Med. Chem. Lett. 14, 3539-3543.

41. Shigemori, H., Kasai, Y., Komatsu, K., Tsuda, M., Mikami, Y. and Kobayashi, J. 2004. Sporiolides A and B, new cytotoxic twelve membered macrolides from a marine derived fungus Cladosporium species. Mar. Drugs. 2, 164169.

42. Lang, G., Mitova, M.I., Ellis, G., van der Sar, S., Phipps, R.K., Blunt, J.W., Cummings, N.J., Cole, A.L.J. and Munro, M.H.G. 2006. Bioactivity profiling using HPLC/microtiterplate analysis: application to a New Zealand marine alga derived fungus, Gliocladium sp. J. Nat. Prod. 69, 621-624. doi:10.1021/np0504917

43. Wang, S., Li, X.M., Teuscher, F., Li, D.L., Diesel, A., Ebel, R., Proksch, P. and Wang, B.G. 2006b. Chaetopyranin, a benzaldehyde derivative, and other related metabolites from Chaetomium globosum, an endophytic fungus derived from the marine red alga Polysiphonia urceolata. J. Nat. Prod. 69 , 1622-1625. doi:10.1021/np060248n

44. Abdel-Lateff, A. 2008. Chaetominedione, a new tyrosine kinase inhibitor isolated from the algicolous marine fungus Chaetomium sp. Tetrahedron Lett. 49, 6398-6400. doi:10.1016/j.tetlet.2008.08.064

45. Krick, A., Kehraus, S., Gerhäuser, C., Klimo, K., Nieger, M., Maier, A., Fiebig, H.H., Atodiresei, I., Raabe, G., Fleischhauer, J. and König, G.M. 2007. Potential cancer chemopreventive in vitro activities of monomeric xanthone derivatives from the marine algicolous fungus Monodictys putredinis. J. Nat. Prod. 70, 353-360.

46. Greve, H., Schupp, P.J., Eguereva, E., Kehraus, S., Kelter, G., Maier, A., Fiebig, H.H. and König, G.M. 2008. Apralactone A and a new stereochemical class of curvularins from the marine derived fungus Curvularia sp. Eur. J. Org. Chem. 2008. doi:10.1002/ejoc.200800522

47. Gao, S.S., Li, X.M., Du, F.Y., Li, C.S., Proksch, P. and Wang, B.G. 2010. Secondary metabolites from a marine derived endophytic fungus Penicillium chrysogenum QEN24S. Mar. Drugs. 9, 59-70. doi:10.3390/md9010059

48. Haroon, M.H., Premaratne, S.R., Choudhry, M.I. and Dharmaratne, H.R.W. 2013. A new $\beta$-glucuronidase inhibiting butyrolactone from the marine endophytic fungus Aspergillus terreus. Nat. Prod. Res. 27, 1060-1066. doi:10.1080/14786419.2012.708659

49. Zhang, P., Li, X.M., Wang, J.N., Li, X. and Wang, B.G. 2015a. New butenolide derivatives from the marine derived fungus Paecilomyces variotii with DPPH radical scavenging activity. Phytochem. Lett. 11, 85-88.

50. Frenz, J.L., Kohl, A.C. and Kerr, R.G. 2004. Marine natural products as therapeutic agents: part 2. Expert Opin. Ther. Pat. 14, 17-33. doi:10.1517/13543776.14.1.17 
51. Pontius, A., Krick, A., Kehraus, S., Foegen, S.E., Müller, M., Klimo, K., Gerhäuser, C. and König, G.M. 2008a. Noduliprevenone: a novel heterodimeric chromanone with cancer chemopreventive potential. Chemistry. 14, 98609863. doi:10.1002/chem.200801574

52. Pontius, A., Krick, A., Mesry, R., Kehraus, S., Foegen, S.E., Müller, M., Klimo, K., Gerhäuser, C. and König, G.M. 2008b. Monodictyochromes A and B, dimeric xanthone derivatives from the marine algicolous fungus Monodictys

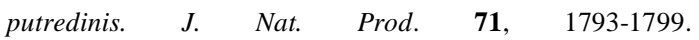
doi:10.1021/np800392w

53. Zhu, T.J., Du, L., Hao, P.F., Lin, Z.J. and Gu, Q.Q. 2009. Citrinal A, a novel tricyclic derivative of citrinin, from an algicolous fungus Penicillium sp. i11. Chin. Chem. Lett. 20, 917-920. doi:10.1016/j.cclet.2009.03.009

54. de Felício, R., Pavão, G.B., de Oliveira, A.L.L., Erbert, C., Conti, R., Pupo, M.T., Furtado, N.A.J.C., Ferreira, E.G., Costa Lotufo, L.V., Young, M.C.M., Yokoya, N.S. and Debonsi, H.M. 2015. Antibacterial, antifungal and cytotoxic activities exhibited by endophytic fungi from the Brazilian marine red alga Bostrychia tenella (Ceramiales). Rev. Bras. Farmacogn. 25, 641-650. doi:10.1016/j.bjp.2015.08.003

55. Nagle, D.G., Zhou, Y.-D., Mora, F.D., Mohammed, K.A. and Kim, Y.-P. 2004. Mechanism targeted discovery of antitumor marine natural products. Curr. Med. Chem. 11, 1725-1756.

56. Almeida, C., Eguereva, E., Kehraus, S., Siering, C. and König, G.M. 2010. Hydroxylated sclerosporin derivatives from the marine derived fungus Cadophora malorum. J. Nat. Prod. 73, 476-478. doi:10.1021/np900608d

57. Wu, B., Wu, X., Sun, M. and Li, M. 2013. Two novel tyrosinase inhibitory sesquiterpenes induced by $\mathrm{CuCl}_{2}$ from a marine derived fungus Pestalotiopsis sp. Z233. Mar. Drugs. 11, 2713-2721. doi:10.3390/md11082713

58. Gao, S.S., Li, X.M., Zhang, Y., Li, C.S. and Wang, B.G. 2011. Conidiogenones $\mathrm{H}$ and $\mathrm{I}$, two new diterpenes of cyclopiane class from a marine derived endophytic fungus Penicillium chrysogenum QEN24S. Chem. Biodivers. 8, 1748-1753. doi: $10.1002 / \mathrm{cbdv} .201000378$

59. Sun, H.F., Li, X.M., Meng, L., Cui, C.M., Gao, S.S., Li, C.S., Huang, C.G. and Wang, B.G. 2012. Asperolides A-C, tetranorlabdane diterpenoids from the marine alga derived endophytic fungus Aspergillus wentii EN48. J. Nat. Prod.75, 148-152. doi: $10.1021 / \mathrm{np} 2006742$

60. Ishino, M., Kiyomichi, N., Takatori, K., Sugita, T., Shiro, M., Kinoshita, K., Takahashi, K. and Koyama, K. 2010. Phomactin I, 13-epi-Phomactin I, and Phomactin J, three novel diterpenes from a marine derived fungus. Tetrahedron. 66, 2594-2597. doi:10.1016/j.tet.2010.02.040

61. Huang, Y.F., Li, L.H., Tian, L., Qiao, L., Hua, H.M. and Pei, Y.H. 2006. Sg1714, a novel isocoumarin from a marine fungus Alternaria tenuis Sg171. J. Antibiot. 59, 355-357. doi:10.1038/ja.2006.50

62. Li, X., Li, X.M., Xu, G.M., Li, C.S. and Wang, B.G. 2014. Antioxidant metabolites from marine alga derived fungus Aspergillus wentii EN48. Phytochem. Lett. 7, 120-123. doi:10.1016/j.phytol.2013.11.008
63. Fang, W., Lin, X., Wang, J., Liu, Y., Tao, H. and Zhou, X. 2016. Asperpyrone-type bis-naphtho- $\gamma$-pyrones with COX2-inhibitory activities from marine-derived fungus Aspergillus niger. Molecules. 21, 941.

64. Liu, X.H., Tang, X.Z., Miao, F.P. and Ji, N.Y. 2011. A new pyrrolidine derivative and steroids from an algicolous Gibberella zeae strain. Nat. Prod. Commun. 6, 1243-1246.

65. Cui, C.M., Li, X.M., Li, C.S., Sun, H.F., Gao, S.S. and Wang, B.G. 2009. Benzodiazepine alkaloids from marine derived endophytic fungus Aspergillus ochraceus. HCA. 92, 1366-1370. doi:10.1002/hlca.200900084

66. Zhang, L., Long, Y., Lei, X., Xu, J., Huang, Z., She, Z., Lin, Y., Li, J. and Liu, L. 2016. Azaphilones isolated from an alga-derived fungus Penicillium sp. ZJ-27. Phytochem. Lett. 18, 180-186. doi:10.1016/j.phytol.2016.10.010

67. Tsuda, M., Kasai, Y., Komatsu, K., Sone, T., Tanaka, M., Mikami, Y. and Kobayashi, J. 'ichi. 2004. Citrinadin A, a novel pentacyclic alkaloid from marine derived fungus Penicillium citrinum. Org. Lett. 6, 3087-3089. doi:10.1021/ol048900y

68. Mugishima, T., Tsuda, M., Kasai, Y., Ishiyama, H., Fukushi, E., Kawabata, J., Watanabe, M., Akao, K. and Kobayashi, J. 'ichi. 2005. Absolute stereochemistry of citrinadins A and B from marine derived fungus. J. Org. Chem. 70, 9430-9435. doi:10.1021/jo051499o

69. Lin, A.Q., Du, L., Fang, Y.C., Wang, F.Z., Zhu, T.J., Gu, Q.Q. and Zhu, W.M. 2009. Iso- $\alpha$-cyclopiazonic acid, a new natural product isolated from the marine derived fungus Aspergillus flavus CF3. Chem. Nat. Compd. 45, 677. doi:10.1007/s1060000994338

70. Izumikawa, M., Hashimoto, J., Takagi, M. and Shinya, K. 2010. Isolation of two new terpeptin analogs JBIR81 and JBIR82 from a seaweed derived fungus, Aspergillus sp. SpD081030G1f1. J. Antibiot. 63, 389-391. doi:10.1038/ ja.2010.57

71. Cui, C.M., Li, X.M., Li, C.S., Proksch, P. and Wang, B.G. 2010a. Cytoglobosins A-G, cytochalasans from a marine derived endophytic fungus, Chaetomium globosum QEN14. J. Nat. Prod. 73, 729-733. doi:10.1021/np900569t

72. Elsebai, M.F., Nazir, M., Kehraus, S., Egereva, E., Ioset, K.N., Marcourt, L., Jeannerat, D., Gütschow, M., Wolfender, J.L. and König, G.M. 2012. Polyketide skeletons from the marine alga derived fungus Coniothyrium cereale. Eur. J. Org. Chem. 2012, 6197-6203. doi:10.1002/ejoc.201200700

73. König, G.M. and Wright, A.D. 1996. Marine natural products research: current directions and future potential. Planta Med. 62, 193-211. doi:10.1055/s-2006-957861

74. Yanagihara, M., SasakiTakahashi, N., Sugahara, T., Yamamoto, S., Shinomi, M., Yamashita, I., Hayashida, M., Yamanoha, B., Numata, A., Yamori, T. and Andoh, T. 2005. Leptosins isolated from marine fungus Leptoshaeria species inhibit DNA topoisomerases I and/or II and induce apoptosis by inactivation of Akt/protein kinase B. Cancer Sci. 96, 816824. doi:10.1111/j.13497006.2005.00117.x 
75. Cueto, M., Jensen, P.R. and Fenical, W. 2000. Nmethylsansalvamide, a cytotoxic cyclic depsipeptide from a marine fungus of the genus fusarium. Phytochemistry. 55, 223-226.

76. Li, Y., Li, X., Kim, S.K., Kang, J.S., Choi, H.D., Rho, J.R. and Son, B.W. 2004. Golmaenone, a new diketopiperazine alkaloid from the marine derived fungus Aspergillus sp. Chem. Pharm. Bull. 52, 375-376.

77. Komatsu, K., Shigemori, H. and Kobayashi, J. 2001. Dictyonamides A and B, new peptides from marine derived fungus. J. Org. Chem. 66, 6189-6192.

78. Elsebai, M.F., Kehraus, S. and König, G.M. 2013. Caught between triterpene and steroid metabolism: $4 \alpha$-carboxylic pregnane derivative from the marine alga derived fungus Phaeosphaeria spartinae. Steroids. $\mathbf{7 8}, \quad 880-883$. doi:10.1016/j.steroids.2013.05.003

79. Cui, C.M., Li, X.M., Meng, L., Li, C.S., Huang, C.G. and Wang, B.G. 2010b. 7-norergosterolide, a pentalactone containing norsteroid and related steroids from the marine derived endophytic Aspergillus ochraceus EN31. J. Nat. Prod. 73, 1780-1784. doi:10.1021/np100386q

80. Yang, R., Li, C., Lin, Y., Peng, G., She, Z. and Zhou, S. 2006. Lactones from a brown alga endophytic fungus (no ZZF36) from the South China Sea and their antimicrobial activities. Bioorganic Med. Chem. Lett. 16, 4205-4208. doi:10.1016/j.bmcl.2006.05.081

81. Li, Y., Li, X., Lee, U., Kang, J.S., Choi, H.D. and Sona, B.W. 2006. A new radical scavenging anthracene glycoside, asperflavin ribofuranoside, and polyketides from a marine isolate of the fungus Microsporum. Chem. Pharm. Bull. 54, 882-883. doi:10.1248/cpb.54.882

82. Pontius, A., Krick, A., Kehraus, S., Brun, R. and König, G.M. 2008. Antiprotozoal activities of heterocyclic substituted xanthones from the marine derived fungus Chaetomium sp. J. Nat. Prod. 71, 1579-1584. doi:10.1021/np800294q

83. Zhang, Y., Li, X.M. and Wang, B.G. 2012. Anthraquinone derivatives produced by marine derived fungus Aspergillus versicolor EN7. Biosci. Biotechnol. Biochem. 76, 1774-1776. doi:10.1271/bbb.120047

84. Hawas, U.W., El-Beih, A.A. and El-Halawany, A.M. 2012. Bioactive anthraquinones from endophytic fungus Aspergillus versicolor isolated from red sea algae. Arch. Pharm. Res. 35, 1749-1756. doi:10.1007/s122720121006x

85. Tarman, K., Palm, G.J., Porzel, A., Merzweiler, K., Arnold, N., Wessjohann, L.A., Unterseher, M. and Lindequist, U. 2012. Helicascolide C, a new lactone from an Indonesian marine algicolous strain of Daldinia eschscholzii (Xylariaceae, Ascomycota). Phytochem. Lett. 5, 83-86. doi:10.1016/j.phytol.2011.10.006

86. Sun, R.R., Miao, F.P., Zhang, J., Wang, G., Yin, X.L. and Ji, N.Y. 2013. Three new xanthone derivatives from an algicolous isolate of Aspergillus wentii. Magn. Reson. Chem. 51, 65-68. doi:10.1002/mrc.3903
87. Dai, J., Krohn, K., Flörke, U., Pescitelli, G., Kerti, G. and Papp T. 2010. Curvularin-type metabolites from the fungus Curvularia sp. isolated from a marine algae. Eur. J. Org. Chem. 6928-6937.

88. Zhang, Y., Li, X.M., Wang, C.Y. and Wang, B.G. 2007a. A new naphthoquinoneimine derivative from the marine algalderived endophytic fungus Aspergillus niger EN-13. Chin. Chem. Lett. 18, 951-953.

89. Li, H.-L., Li, X.-M., Liu, H., Meng, L.-H. and Wang, B.-G. 2016. Two new diphenylketones and a new xanthone from Talaromyces islandicus EN-501, an endophytic fungus derived from the marine red alga Laurencia okamurai. Mar. Drugs. 14, 223. doi:10.3390/md14120223

90. Osterhage, C., König, G.M., Höller, U. and Wright, A.D. 2002. Rare sesquiterpenes from the algicolous fungus Drechslera dematioidea. J. Nat. Prod. 65, 306-313.

91. Krohn, K., Dai, J., Flörke, U., Aust, H.J., Dräger, S. and Schulz, B. 2005. Botryane metabolites from the fungus Geniculosporium sp. isolated from the marine red alga Polysiphonia. J. Nat. Prod. 68, 400-405. doi:10.1021/ np0498206

92. Liu, X.H., Miao, F.P., Li, X.D., Yin, X.L. and Ji, N.Y. 2012a. A new sesquiterpene from an endophytic Aspergillus versicolor strain. Nat. Prod. Commun. 7, 819-820.

93. Liu, X.H., Miao, F.P., Qiao, M.F., Cichewicz, R.H. and Ji, N.Y. 2012b. Terretonin, ophiobolin, and drimane terpenes with absolute configurations from an algicolous Aspergillus ustus. RSC Adv. 3, 588-595. doi:10.1039/C2RA22701K

94. Qiao, M.F., Ji, N.Y., Liu, X.H., Li, K., Zhu, Q.M. and Xue, Q.Z. 2010. Indoloditerpenes from an algicolous isolate of Aspergillus oryzae. Bioorg. Med. Chem. Lett. 20, 5677-5680. doi:10.1016/j.bmcl.2010.08.024

95. Gao, S.S., Li, X.M., Zhang, Y., Li, C.S. and Wang, B.G. 2011a. Conidiogenones $\mathrm{H}$ and I, two new diterpenes of cyclopiane class from a marine-derived endophytic fungus Penicillium chrysogenum QEN-24S. Chem. Biodivers. 8, 1748-1753.

96. Gao, S.S., Li, X.M., Du, F.Y., Li, C.S., Proksch, P. and Wang, B.G. 2011b. Secondary metabolites from a marinederived endophytic fungus Penicillium chrysogenum QEN24S. Mar. Drugs. 9: 59-70.

97. Sun, H., Li, X., Meng, L., Cui, C., Gao, S., Li, C., Huang, C. and Wang, B. 2012. Asperolides A-C, tetranorlabdane diterpenoids from the marine alga-derived endophytic fungus Aspergillus wentii EN-48. J. Nat. Prod. 75, 148-152.

98. Liu, H., Li, X.-M., Liu, Y., Zhang, P., Wang, J.-N. and Wang, B.-G. 2016. Chermesins A-D: meroterpenoids with a drimane-type spirosesquiterpene skeleton from the marine algal-derived endophytic fungus Penicillium chermesinum EN-480. J. Nat. Prod. 79, 806-811. doi:10.1021/ acs.jnatprod.5b00893

99. Liang, X.-R., Miao, F.-P., Song, Y.-P., Guo, Z.-Y. and Ji, N.Y. 2016. Trichocitrin, a new fusicoccane diterpene from the marine brown alga-endophytic fungus Trichoderma citrinoviride cf-27. Nat. Prod. Res. 30, 1605-1610. doi:10.1080/14786419.2015.1126264 
100. Yang, G., Sandjo, L., Yun, K., Leutou, A.S., Kim, G.D., Choi, H.D., Kang, J.S., Hong, J. and Son, B.W. 2011. Flavusides A and B, antibacterial cerebrosides from the marine derived fungus Aspergillus flavus. Chem. Pharm. Bull. 59, 1174-1177.

101. Li, X., Li, X.M., Zhang, P. and Wang, B.G. 2015. A new phenolic enamide and a new meroterpenoid from marine alga derived endophytic fungus Penicillium oxalicum EN290. J. Asian Nat. Prod. Res. 17, 1204-1212. doi:10.1080/10286020.2015.1117454

102. Silva, E.D.De, Geiermann, A., Mitova, M.I., Kuegler, P., Blunt, J.W. and Cole, A.L.J. 2009. Isolation of 2-pyridone alkaloids from a New Zealand marine-derived Penicillium species. J. Nat. Prod. 72: 477479.

103. Miao, F., Li, X., Liu, X., Cichewicz, R.H. and Ji, N. 2012. Secondary metabolites from an algicolous Aspergillus versicolorstrain. Mar. Drugs. 10, 131-139.

104. Zhang, Y., Li, X.M. and Wang, B.G. 2007C. Nigerasperones A-C, new monomeric and dimeric naphtho-g-pyrones from a marine alga-derived endophytic fungus Aspergillus niger EN-13. J. Antibiot. 60: 204-210.

105. Osterhage, C., Kaminsky, R., König, G.M. and Wright, A.D. 2000. Ascosalipyrrolidinone A, an antimicrobial alkaloid, from the obligate marine fungus Ascochyta salicorniae. J. Org. Chem. 65, 6412-6417. doi:10.1021/jo000307g

106. Zhuravleva, O.I., Afiyatullov, S.S., Denisenko, V.A., Ermakova, S.P., Slinkina, N.N., Dmitrenok, P.S. and Kim, N.Y. 2012. Secondary metabolites from a marine derived fungus Aspergillus carneus Blochwitz. Phytochemistry. 80, 123-131. doi:10.1016/j.phytochem.2012.05.008

107. Zhang, P., Mándi, A., Li, X.M., Du, F.Y., Wang, J.N., Li, X., Kurtán, T. and Wang, B.G. 2014. Varioxepine A, a 3-Hoxepinecontaining alkaloid with a new oxacage from the marine algal derived endophytic fungus Paecilomyces variotii. Org. Lett. 16, 4834-4837. doi:10.1021/ol502329k

108. Zhang, P., Li, X.M., Wang, J.N. and Wang, B.G. 2015 b. Oxepine containing diketopiperazine alkaloids from the algal derived endophytic fungus Paecilomyces variotii EN291. HCA. 98, 800-804. doi:10.1002/hlca.201400328

109. Du, F.Y., Li, X.M., Song, J.Y., Li, C.S. and Wang, B.G. 2014. Anthraquinone derivatives and an orsellinic acid ester from the marine alga derived endophytic fungus Eurotium cristatum EN220. HCA. 97, 973-978. doi:10.1002/ hlca.201300358

110. Yang, H., fang, L.I. and Naiyun, J.I. 2016. Alkaloids from an algicolous strain of Talaromyces sp. Chin. J. Oceanol. Limnol. 1-5.
111. Du, F.-Y., Li, X., Li, X.-M., Zhu, L.-W. and Wang, B.-G. 2017. Indolediketopiperazine alkaloids from Eurotium cristatum EN-220, an endophytic fungus isolated from the marine alga Sargassum thunbergii. Mar. Drugs. 15, 24. doi: $10.3390 / \mathrm{md} 15020024$

112. Gao, S.S., Li, X.M., Li, C.S., Proksch, P. and Wang, B.G. 2011. Penicisteroids A and B, antifungal and cytotoxic polyoxygenated steroids from the marine alga derived endophytic fungus Penicillium chrysogenum QEN24S. Bioorganic Med. Chem. Lett. 21, 2894-2897. doi:10.1016/j.bmcl.2011.03.076

113. Liu, X.F., Miao, F., Liang, X. and Ji, N. 2014. Ergosteroid derivatives from an algicolous strain of Aspergillus ustus. Nat. Prod. Lett. 28, 1182-1186.

114. Sebastianes, F.L.S., Cabedo, N., Aouad, N.E., Valente, A.M.M.P., Lacava, P.T., Azevedo, J.L., Pizzirani-Kleiner, A.A. and Cortes, D. 2012. 3-hydroxypropionic acid as an antibacterial agent from endophytic fungi Diaporthe phaseolorum. Curr. Microbiol. $\quad 65$, 622-632. doi:10.1007/s0028401202064

115. Hawas, U.W., El-Halawany, A.M. and Ahmed, E.F. 2013. Hepatitis C virus NS3NS4A protease inhibitors from the endophytic Penicillium chrysogenum isolated from the red alga Liagora viscida. Z. Naturforsch., C, J. Biosci. 68, 355366.

116. Zhang, Y., Wang, S., Li, X.M., Cui, C.M., Feng, C. and Wang, B.G. 2007b. New sphingolipids with a previously unreported 9-methyl-C20-sphingosinemoiety from a marine algicolous endophytic fungus Aspergillus niger EN-13. Lipids. 42, 759-764.

117. Puri, S. C., Nazir, A., Chawla, R., Arora, R., Riyaz-ul-Hasan, S., Amna, T., Ahmed, B., Verma, V., Singh, S., Sagar, R., Sharma, A., Kumar, R., Sharma, R.K., and Qazi, G. N. 2006. The endophytic fungus Trametes hirsuta as a novel alternative source of podophyllotoxin and related aryl tetralin lignans. J. Biotechnol. 122, 494-510. doi:10.1016/j.jbiotec. 2005.10.015

118. Song, Y. C., Huang, W. Y., Sun, C., Wang, F. W., and Tan, R. X. 2005. Characterization of graphislactone A as the antioxidant and free radical-scavenging substance from the culture of Cephalosporium sp. IFB-E001, an endophytic fungus in Trachelospermum jasminoides. Biol. Pharm. Bull. 28, 506-509. doi:10.1248/bpb.28.506

119. Lee, J. C., Lobkovsky, E., Pliam, N. B., Strobel, G., and Clardy, J. 1995. Subglutinols A and B: immunosuppressive compounds from the endophytic fungus Fusarium subglutinans. J. Org. Chem. 60, 7076-7077. 